\title{
IMPACT EROSION OF TERRESTRIAL PLANETARY ATMOSPHERES
}

\section{Thomas J. Ahrens}

Lindhurst Laboratory of Experimental Geophysics, Seismological Laboratory 252-21, California Institute of Technology, Pasadena, CA 91125 USA

Shortened title: Atmospheric Erosion

Send proofs to: Dr. Thomas J. Ahrens, Seismological Laboratory 252-21, California Institute of Technology, Pasadena, CA 91125 (Telephone 818-356-6906)

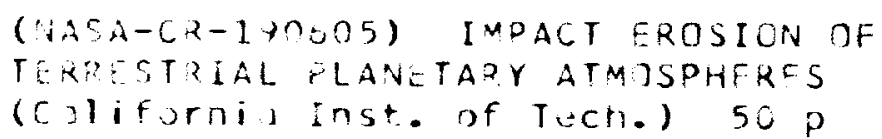

$N 92-30921$

Unclas

$03 / ? 1 \quad 0112089$ 


\section{TABLE OF CONTENTS}

1. INTRODUCTION_ 3

2. PLANET FORMING MATERIALS___ 4

1) Planetesimals similar to meteorites___ 4

2) Cometary Planetesimals _ 5

3) Solar Nebulae Gas _ 5

3. TERRESTRIAL PLANETARY VOLATILES 5

4. SIZE DISTRIBUTION OF PLANETESIMALS 6

5. PLANETESIMAL IMPACT VELOCITIES__

6. COACCRETION OF PLANETARY ATMOSPHERES 9

7. ATMOSPHERIC EROSION BY IMPACT CRATERING_ 12

8. ATMOSPHERIC BLOW-OFF BY GLANT IMPACTS 15

\begin{tabular}{ll} 
ACKNOWLEDGMENTS & 21 \\
\hline
\end{tabular} 


\section{INTRODUCTION}

The idea that planetary atmospheres can erode as a result of impact, and thus lose mass along with solid and molten high velocity ejecta during accretional infall of planetesimals follows from such early thoughtful works as that of Arrhenius et al [1974], Benlow and Meadows [1977], Ringwood [1979], and Cameron [1983]. Ahrens et al [1989] describe how planetary impact accretion (and impact erosion) concepts lead naturally, from the idea that atmospheres form and erode, during planetary growth.

The theory of planetary system formation from a disc of gas, and later, gas and dust, corotating around the proto-sun, is described by Safronov [1969], Wetherill [1980], and Kaula [1979] (Fig. 1). In this model, planets grow as a result of mutual attraction and collision of planetesimals within the early solar nebula. An important step in the wide acceptance of this model was the theoretical work of Goldreich \& Ward [1973] who quantitatively showed that condensed material from a solar nebula would grow into the kmsized planetesimals hypothesized by Safronov. Recently, very strong support for the Safronov-Wetherill-Kaula scenario of planetary growth via mutual collision has come from discovery using infrared, optical, and radio imagery of disc-shaped circumstellar gas and dust clouds around T-Tauri stars (e.g. HL Tauri and R Monocerotis) [Beckwith et al, 1986; Sargent \& Beckwith, 1987] and main sequence stars (e.g. $\beta$-Pictoris) [Smith \& Terrile, 1984] (Fig. 2). Although the gaseous rotating discs around T-Tauri stars have been imaged via microwave radio interferometry and infrared techniques, it has not been demonstrated that these discs contain accreting planetesimals. Recent observations of the ${ }^{13} \mathrm{CO}$ emission spectra and the blackbody thermal emission from the circumstellar gas disc around HL Tauri indicate that this disc extends out to a radius of $2000 \mathrm{AU}$, but is less than 380 AU thick. Moreover, the spectra are consistent with the gas and dust moving in bound orbits around this star [Sargent \& Beckwith, 1992].

I review current ideas about the nature of the planetesimals --composition, size 
distribution, and the planetary encounter velocity. Previous papers on accretion and erosion of planetary atmospheres as a result of multiple impacts are reviewed. Finally, the effects of blowing off a substantial fraction of the atmosphere from a terrestrial planet due to a single giant body impact are discussed.

\section{PLANET FORMING MATERIALS}

The planets and minor objects in the solar system appear to have accreted from the following three components:

1) Planetesimals similar to meteorites.

The constitution of the terrestrial planets suggest that they accreted largely from planetesimals with a range of composition including primitive objects, such as, $\mathrm{Cl}$ chondrites, as well as objects similar to differentiated metal and silicate meteorites. The linear relation in Fig. 3 indicates how similar the major element composition of the terrestrial planets are to the sun, while Fig. 4 illustrates that the noble gas abundance patterns of terrestrial planets are similar to those of primitive meteorites such as $\mathrm{Cl}$ chondrites. Meteorites are often taken to be typical of the planetesimals existing within the inner zone of the solar nebula from which the terrestrial planets accreted. The vestiges of the planetesimals of the inner solar system are believed to be the asteroids. We presumably sample these objects via meteorites that fall on the Earth. Some of the planetesimals which formed the terrestrial planets were also probably similar in composition to the present comets. Largely because of the gravitational perturbation due to the large masses of Jupiter and Saturn, comets can achieve highly eccentric orbits which, near perihelion, result in collision with the terrestrial planets. Shoemaker et al [1990] estimated that some $30 \%$ of the recent impactor flux on the earth may be cometary. How much of this flux has provided the volatile budgets of the terrestrial planets is presently unclear (e.g. Grinspoon 
\& Lewis [1988] and Donahue \& Hodges [1992]) because of the similarity of the cometary non-volatile major element inventory with that of the $\mathrm{Cl}$ chondrites [Jessberger et al, 1989].

\section{2) Cometary Planetesimals}

In contrast to the silicate and iron-rich planetesimals, the planetesimals that made up of the cores of the giant outer planets - Jupiter and Saturn and to a lesser degree, Neptune and Uranus, and possibly, Pluto, were ice-rich. Their remnants are now associated with comets. The generally smaller size ( $<10 \mathrm{~km}$ diameter) of the comets (relative to asteroids) suggest that in the outer solar system the density of matter in the solar nebula was never great enough for the ice-rich planetesimals to experience substantial mutual gravitational attraction, resulting in impact accretion.

\section{3) Solar Nebulae Gas}

In contrast to terrestrial planets, Jupiter and Satum, and to a lesser degree, Uranus and Neptune appear to have, after building up their initial planetary core from ice, silicate, and carbon-rich objects, gravitationally captured large quantities of solar nebular gases. The large planets thus retain a large solar-like reservoir of $\mathrm{H}_{2}$ and He. Pollack \& Bodenheimer [1989] suggested that the ratio of carbon to hydrogen in the atmospheres which dramatically increases in the planets, in the order: Jupiter, Saturn, Uranus, and Neptune, directly reflects a decreasing budget of accreted solar nebular hydrogen.

\section{TERRESTRIAL PLANETARY VOLATILES}

On the basis of the general similarity of the noble gas abundances of the terrestrial planet atmospheres to the noble gas component which was processed within planetesimals before their accretion, $I$ infer that terrestrial planet atmospheres originated from planetesimals similar to primitive meteorites. Cometary-like planetesimals may have also 
contributed to the terrestrial planet volatile inventory. Notably, the thermal and gravitational evolution of the planets are distinctly different, however, their relative noble gas inventories are similar. The solid phases containing noble gases presumably are still present in the asteroids and demonstratively occur in meteorites. Small meteorites impact at sufficiently slow terminal velocity that the noble gases are not released upon impact. They still contain the complement of noble gases which were present $4.6 \mathrm{Gyr}$ ago prior to the planet-forming epoch (Fig. 2). Moreover, the two key mass-selective, gas-loss mechanisms (e.g. Hunten et al [1989]) for atmospheric escape from planets, Jeans loss and hydrodynamic escape, predict abundance patterns, starting from a solar noble gas pattern, which are quite different from those in the atmospheres of Mars, Earth and Venus.

\section{SIZE DISTRIBUTION OF PLANETESIMALS}

In Safronov's [1969] theory of planetary accretion, a simple power law distribution of the differential number of objects, $d N$, which occurred in a mass range, $d m$ is assumed

$$
\mathrm{dN}=\mathrm{m}-\mathbf{q} \mathrm{dm}
$$

where $1<q<2$.

For very energetic collisions, more mass becomes concentrated in the fines, which implies that $q>2$. As pointed out by Greenberg [1989] when $q=2$, each size range contains exactly the same total mass. Observations of the present asteroids, which presumably have collided and reaccreted over their 4.6 Gyr lifetime show a mass distribution close to $q=2$. However, statistical studies of accretion models indicate that values closer to $q=1.5$ occurred in the past which implies that most of the mass of the impactor population occurred in the largest planetesimals. Weak constraints on the value of $q$ for impactors in the solar system come from the distribution of crater sizes on ancient cratered terranes, such as the highlands of the Moon. If a planet with a current mass, $M$, is 
accreting a veneer of material, of mass, $\delta \mathrm{M}$, the mass of the largest impactor, $\mathrm{m}_{1}$, is obtained by integrating:

$$
\delta M=\int_{0}^{m_{1}} m N(m) d m
$$

and assuming that Eq. 1 is valid up to the size of the largest bodies, for $q<2$ [Greenberg, 1989; Wetherill, 1985; Wetherill \& Stewart, 1989]

$$
m_{1}=\delta M(4-2 q) /(3-q)
$$

Thus, for example, if the accretion of $10 \%$ of the earth, $\delta \mathrm{M}=6 \times 10^{26} \mathrm{~g}$ and $\mathrm{q}=1.5$, then $\mathrm{m}_{1}=4 \times 10^{26} \mathrm{~g}$, or $\mathrm{m}_{1}$ has a radius of $2.6 \times 10^{8} \mathrm{~cm}$, which is similar to Mar's radius. Thus we need to consider the effect of impact on planets of very substantial projectiles having radii, perhaps $10^{2}$ times the scale height of the planetary atmosphere.

\section{PLANETESIMAL IMPACT VELOCITIES}

As shown in Fig. 1, the major element of motion of the planetesimals in ideal circular Keplerian circular orbits, around the sun, is velocity, $v_{s}$, which, in the solar reference frame is given by

$$
v_{s}=\sqrt{\mathrm{GM}_{\theta} / \mathrm{r}}
$$

where $G$ is the gravitational constant $\left(6.67 \times 10^{-8} \mathrm{dyn}-\mathrm{cm}^{2} / \mathrm{g}^{2}\right.$ or $\left.6.7 \times 10^{-11} \mathrm{~nm}^{2} / \mathrm{kg}^{2}\right), \mathrm{M}_{\Theta}$ is the solar mass and $\mathrm{r}$ is the distance of the planetesimal from the sun's center. Even for perfectly circular planar orbits, two objects at slightly different solar distances, initially will differ in velocity. Hence, the object closest to the sun, $m_{1}$, will move in at a slightly higher speed, passing an object, $m_{2}$, which is further from the sun. Gravitational interaction occurs in this two-body encounter, such that the object, $\mathbf{m}_{1}$, will experience a radial velocity increase given by 


$$
\delta v=m_{2} v_{d}\left(m_{1}+m_{2}\right) E
$$

Here, $E$, is an encounter parameter which depends on the geometry and the relative masses of the objects. Continual gravitational interaction of adjacent objects gives rise to increasing orbital eccentricities, as well as inclination of the orbits. These in turn give rise to collisions which tend to damp out the velocity and orbital perturbations such as described by Eqn. 4 . On average, for planetesimals relative to one another in orbit in a corotating disc of particles, their encounter velocities will be $v_{\infty}$. Safronov pointed out that the largest particles with an escape velocity, $v_{e}$, will have encounter velocities:

$$
v_{\infty}=v_{d} \sqrt{2 \theta}
$$

Here the local escape velocity is

$$
v_{e}=\sqrt{2 G m_{p} / R_{p}}=\sqrt{2 R_{p} g}
$$

The result depends critically on $\mathrm{q}<2$. Otherwise, $v_{\infty}$ will be drastically reduced by multiple encounters with the smallest particles.

The impact velocity of a planetesimal is therefore:

$$
v_{i}=\sqrt{\left(v_{e}^{2}+v_{\infty}^{2}\right)}
$$

where $m_{p}$ is the mass of the largest planetesimal in a region, $R_{p}$ is the largest planetesimal radius, and $\mathrm{g}$ is its gravitational acceleration. Here, $\theta$, the Safronov parameter, is usually taken to be about 4 or 5 . Eq. 7 implies that as planets grow by accretion, the velocity is always somewhat greater than the planetary escape velocity. Moreover, Eq. $2 \mathrm{a}$ indicates that as planets grow, so does the mass of the planetesimals which impact their surface. These considerations have led to efforts to understand the essential physics of large body impacts on the terrestrial planets by Benz et al $[1989 ; 1986 ; 1987 ; 1988]$ and Kipp \& Melosh [1986]. 


\section{COACCRETION OF PLANETARY ATMOSPHERES}

Although Lange \& Ahrens [1982b] suggested that the impact-induced dehydration of water-bearing minerals in planetesimals such as serpentine $\mathrm{Mg}_{3} \mathrm{Si}_{2} \mathrm{O}_{5}(\mathrm{OH})_{4}$ would produce a largely water-rich atmosphere on the growing planets, it was Abe \& Matsui [1985] who first suggested the possibility that water in this atmosphere, and possibly the dust produced by planetesimal impact could drastically alter the thermal regime on the surface of growing planets (Fig. 5). They assumed that serpentine in planetesimals brought the Earth and the other terrestrial planets their water inventory during their accretion. Once impact pressures of infalling planetesimals exceeded $P=23 \mathrm{GPa}$ (for a porous regolith), the supply of water to the atmosphere was assumed to begin. Abe \& Matsui [1985] estimated the peak shock pressure by

$$
P=p_{0}\left[C_{0}+\left(K^{\prime}+1\right) v_{i} / 8\right] v_{j} / 2
$$

where $\rho_{0}$ and $C_{0}$ are impacting planetesimal initial density and zero-presssure bulk sound velocity, respectively, and $\mathrm{K}^{\prime}$ is the partial derivative of the bulk modulus with respect to pressure. In these atmospheric accretion models the shock pressure is assumed to act on the entire impacting planetesimal and $\mathrm{H}_{2} \mathrm{O}$ is released, as well as lesser amounts of $\mathrm{CO}_{2}$, $\mathrm{NH}_{3}, \mathrm{SO}_{2}$, and other volatiles present in primitive meteorites (e.g. $\mathrm{Cl}$ carbonaceous chondrites). Previous calculations have also included contributions to the atmosphere induced by shock-loading of volatiles already present in the material of the planetary surface layer (e.g., Lange \& Ahrens [1982a]).

Using the experimental value of $23 \mathrm{GPa}$ as the shock pressure required to induce complete water loss for serpentine Lange \& Ahrens [1982b] and Abe \& Matsui [1986] concluded that once the radius of the Earth reached between 0.2 to 0.4 of the present value $\left(R_{\oplus}\right)$, thermal blanketing of the Earth caused by a dense water atmosphere occurred. Matsui \& Abe [1986a] point out that thermal blanketing is a more severe condition than the 
greenhouse effect. In a greenhouse effect, sunlight penetrates through the atmosphere (in the visible) but the thermal energy to be reradiated by the planetary surface in the infrared is trapped because of the infrared opacity of the planetary atmosphere resulting from abundant $\mathrm{CO}_{2}$ and $\mathrm{H}_{2} \mathrm{O}$. Thermal blanketing is more severe, because solar radiation incident on the top of the atmosphere is completely scattered by the fine aerosols and impact ejecta and all the thermal energy is absorbed by the greenhouse gases, $\mathrm{H}_{2} \mathrm{O}$ and $\mathrm{CO}_{2}$. Impact cratering calculations [Ahrens et al, 1989; O'Keefe \& Ahrens, 1977a] demonstrate that for large impactors, 60 to $90 \%$ of the energy of the impact is delivered as internal energy of the near surface material. The major effect of the proto-atmosphere then is to provide an insulating blanket to the flux of heat due to impacts on the surface of the growing planet. These processes are described by the equation

$$
\begin{gathered}
(1+1 / 2 \theta) \frac{G m_{p}}{R_{p}} \dot{m d t}=4 \pi R^{2}\left(F_{a t m}-F_{i}\right) d t \\
+C_{p} \dot{m}_{p}\left(T_{s}-T_{p}\right) d t+C_{p} m_{s} \dot{T}_{s} d t
\end{gathered}
$$

where the left-hand side is the rate of kinetic energy supplied to the surface provided by the impacting planetesimals. The first term on the right is the balance of heat flux of the atmosphere where $F_{a m}$ is the energy flux escaping from the surface to interplanetary space and $F_{i}$ is the energy flux from the interior to the surface layer. The second and third terms are the heat sinks to the planet as a result of heating a larger planetary material of mass $m_{s}$ from planetesimal temperature $T_{p}$ to the higher surface temperature $T_{s}$. For a graybody radiative equilibrium atmosphere

$$
F_{a \operatorname{mon}}=2\left(\sigma T_{s}^{4}-S_{0} / 4\right) /\left[\left(3 k p_{0} / 2 g\right)+2\right]
$$

where $\sigma$ is the Stefan-Boltzmann constant, $S_{0}$ is the solar flux, $\mathbf{k}$ is the absorption coefficient in the atmosphere, and po, is the surface pressure. Thermal blanketing as described by Eqs. (9) and (10), with the reasonable values of the constants chosen by Abe 
\& Matsui [1985; 1986], quickly leads to temperatures above the solidus of crustal (basaltic) rocks (Fig. 6a). Moreover, as more planetesimals impact the planet, the additional water provided begins to dissolve in what is the start of a magma ocean. Abe \& Matsui showed that the surface temperature should be buffered by the solidus of hydrous basalt, $\sim 1500 \mathrm{~K}$, and the mass of $\mathrm{H}_{2} \mathrm{O}$ in the atmosphere is nearly constant at $10^{24} \mathrm{~g}$ (essentially the present surface water budget) (Fig. 6b). They showed that the mass of $\mathrm{H}_{2} \mathrm{O}$ in the atmosphere remains nearly constant (due to a negative feedback effect). This effect can be demonstrated for a small surface temperature increase. This causes an increase in the fraction of molten basalt. That in tum induces additional water to dissolve in the molten silicate. Loss of water from the atmosphere then decreases the effectiveness of atmospheric blanketing and the result is that the small increase in temperature is nullified by the system's negative feedback.

The effectiveness of thermal blanketing of impact energy by the massive protoatmosphere, as well as the feedback effect of water solubility in molten silicates has been independently verified in a study by Zahnle et al [1988].

The termination of the coaccretion of an atmosphere and planet, which was modeled to occur on Earth, Venus and Mars, can occur via three different mechanisms.

1) Abe \& Matsui [1988] suggested that as the accretion rate decreased, the impact energy flux at the base of the atmosphere decreased and gradually solar heating dominated over impact heating. The surface temperature then declined below the melting point of hydrous basalt. With decreasing temperature, the water condensed and formed terrestrial oceans. Oceans may have formed on Venus [Matsui \& Abe, 1986b], however, the larger solar ultraviolet flux gives rise to a enhanced photodisassociation to hydrogen and oxygen in the upper atmosphere and subsequent Jean's escape of a large fraction of the planet's hydrogen inventory gives rise to the presently observed enhancement of the D/H ratio of 
Venus relative to the Earth of $-10^{2}$.

2) Atmospheric loss occurs via multiple impact erosion. This is discussed in the next section.

3) Sudden partial or complete atmospheric loss occurs as a result of a large body impact. This is discussed in Section 7.

\section{ATMOSPHERIC EROSION BY IMPACT CRATERING}

In addition to bringing volatiles to accreting atmospheres, the infall of planetesimals can erode planets and their oceans and atmospheres. For atmosphere-free solid and molten silicate planets, because the mechanical impedance of the impacting planetesimals is, in general, similar to that of a planet, the amount of ejecta which can escape from a planet with a given surface escape velocity depends only on impact velocity (and hence energy per unit mass) [O'Keefe \& Ahrens, 1977b], whereas the net gain or loss of a planetary atmosphere depends on total impact energy. O'Keefe \& Ahrens [1982] calculated the energy partitioning into an atmosphere overlying a planet. They found that upon impact of a planet with projectiles of radii less than the atmospheric scale height, where the ejecta was primarily solid or molten, the amount of energy imparted to the atmosphere by direct passage through the atmosphere was only a few percent. Moreover, very little of the atmosphere achieved upward velocities in excess of the escape velocity. Walker [1986] showed that a very small portion of the atmosphere, shocked by the meteoroid, achieved sufficient enthalpy density to expand to greater than escape velocity. Using a numerical explosion model, Jones \& Kodis [1982] showed that for the Earth, atmospheric explosion energies $>5 \times 10^{26}$ ergs induced significant atmospheric blow-off.

Subsequently, Ahrens \& O'Keefe [1987] and Ahrens et al [1989] employed a model in which they assumed all the energy of the impactor is delivered to the planetary 
surface and applied a theory for the shock acceleration of the atmosphere by an explosion developed by Zel'dovich \& Raizer [1966 Chapt. 12] and Bach et al [1975] (Fig. 7). In this model, the time for atmospheric escape is related to atmospheric density near the Earth's surface, Poo, explosion energy, $E$, and atmospheric scale height, $H$, by

$$
t=C_{1}\left(\rho_{\infty} H^{5 / E}\right)^{1 / 2}
$$

where the constant, $C_{1}$ is approximately equal to 25 . Moreover, the initial atmospheric shock velocity for atmospheric escape is

$$
D=\alpha H / t
$$

where $\alpha \equiv 6$. Assuming the Earth impactor has a velocity, $v_{e}=11 \mathrm{~km} / \mathrm{sec}$ and a strong shock condition exists such that $D \cong 11 \mathrm{~km} / \mathrm{sec}$, Eq. 12 yields $t=4.4 \mathrm{sec}$ and the minimum impact energy calculated from Eq. 11 is $1.9 \times 10^{27}$ ergs. A projectile carrying this energy, if composed of silicate will have a radius of $-0.5 \mathrm{~km}$. The energy, $1.9 \times 10^{27} \mathrm{ergs}$, is somewhat greater (by a factor of 20) than from the numerical calculations of Jones \& Kodis [1982].

For more energetic impacts, (larger impactors) Melosh \& Vickery [1989] and Vickery \& Melosh [1990] developed a simple atmospheric cratering model applicable, for example, on the Earth in the $4.5 \times 10^{27}$ to $9.9 \times 10^{30}$ erg energy range (Fig. 8). For 20 $\mathrm{km} / \mathrm{sec}, 2.7 \mathrm{~g} / \mathrm{cm}^{3}$ impactors, these energies correspond to impactor radii of 0.6 to $7.6 \mathrm{~km}$. They pointed out that for high-velocity planetary impacts, which penetrate the atmosphere, the projectile and an equivalent mass of target become vaporized. The resulting gas plume then expands at a speed greater than the planetary escape velocity and carries with it the overlying planetary atmosphere. A conservative model of the plume expansion gives the mean (mass-averaged) velocity of expansion as

$$
v_{\exp }=\left[2\left(e-h_{\text {vap }}\right)\right]^{1 / 2}
$$


where $e$ is the kinetic energy per unit mass of the impactor and $h_{\text {vep }}$ is the total enthalpy per unit mass, starting at ambient temperature required to vaporize the projectile or target material. For silicate and ice, $h_{\text {vap }}$ is $13 \times 10^{13}$ and $3 \times 10^{13} \mathrm{ergs} / \mathrm{g}$, respectively. The energy per unit mass imparted by the impact-induced shock-wave for like materials is $\mathrm{e}=\mathrm{v}$ $2 / 8$, where we assume the shock particle velocity, $u$ is $v / 2$. It is easy to show that the minimum impact velocity required for the vapor plume to exceed the escape velocity is

$$
v_{m}=\sqrt{8\left(v_{e}^{2} / 2+h_{\text {vap }}\right)}
$$

Atmospheric erosion occurs if the impact-induced momentum of this shock-induced gas when combined with the mass of the overlying atmosphere has sufficient velocity to escape the planet. By assuming a velocity profile in the total expanding gas cloud proposed by Zel'dovich \& Raizer [1966 p. 104] of the form

$$
P(r)=A\left(1-r^{2} / R^{2}\right) \alpha / R^{3}
$$

where $\mathrm{R}$ is the radius of the front of the gas cloud, $\mathrm{r}$ is the radius to a point within the gas cloud, and $A$ and $\alpha$ are determined by assuming conservation of mass and energy above the impact site. Vickery \& Melosh [1990] used a value of $\gamma=9 / 7$ to infer a value of $\alpha=$ 11 and $A=15.4 M_{t}$, where $\gamma$ is the gas cloud's polytropic exponent, and $M_{t}$ is the total mass of the vapor cloud. When the projectile velocity exceeds the minimum impact velocity for atmospheric escape (Eq. 14), escape occurs in atmosphere directly above the impact site. As the impact energy is increased, a cone with increasing angle $\theta$ is ejected (Fig. 9). As impacts become more energetic, the maximum energy in the Vickery-Melosh model corresponds to ejection of an air mass above the tangent plane (Fig. 9) of $3.3 \times 10^{19}$ $g$ (for the case of the Earth) or $6.3 \times 10^{-4}$ of the total atmospheric budget. Vickery and Melosh showed that when the mass of the projectile exceeds the mass of the atmosphere 
above the horizontal tangent, $m_{c}$, all of the atmosphere above a tangent plane to Earth is ejected. Thus when

$$
m \geq m_{c}=H m_{\alpha} / 2 R_{p}
$$

Eq. 16 thus indicates that for smaller $m_{2}$, the mass of the atmosphere, gives rise to a smaller mass, $m_{c}$, which can erode an atmosphere. Table 1 gives $m_{c}$ for the terrestrial planets. Since smaller projectiles are more numerous and thinner atmospheres erode rapidly, Eq. 16 indicates that once an atmosphere starts eroding, erosion is accelerated until the planet is stripped. Fig. 10 shows the maximum atmospheric mass that can be expelled by a spectrum of impactors for three different planets for a given accretion (veneer) mass being added to the planet. Melosh \& Vickery [1989] demonstrated how Mars' atmosphere could have been eroded from an initial surface pressure of 0.7 bars to the present $7 \times 10^{-}$ 3 bars in the first 1.4 Gyr of solar system history.

In conclusion, I note that in the case of the Earth, impact erosion is important only for impacts more energetic than $-10^{27}$ ergs. Thus the Abe-Matsui scenario, which only deals with atmospheric accretion, is relevant if the projectiles impacting the Earth's surface are much smaller in radius than the atmospheric scale height. For projectiles with radii in the km range, the research summarized in this section indicates that impact erosion needs to be taken into account in accretion models of the terrestrial planets.

\section{ATMOSPHERIC BLOW-OFF BY GIANT IMPACTS}

As discussed in Sect. 3, as the planets accreted according to the SafronovWetherill-Kaula scenario, the planetesimal impactors also grew in size and it appears likely that some planetesimals grew to radii in the 2,000 to $3,000 \mathrm{~km}$ range which are comparable to those of the smaller planets (e.g. Mercury and Mars). These considerations have motivated the numerical modeling of large body impacts by Benz et al and Kipp and 


\section{Melosh cited in Section 4.}

Previously, the effects of large body impact on the Earth's atmosphere has only been briefly described by Ahrens [1990]. To calculate the energy, and hence, approximate planetesimal size, such that upon impact the entire planetary atmosphere is blown off, I employ a different approach than previous efforts and consider a shock wave that is entirely propagated within a terrestrial planet as sketched in Fig. 11.

The key calculation is to relate the particle velocity of the solid planet-atmosphere interface, $u_{f s}$, antipodal to a major impact, to the atmospheric free-surface velocity, $v_{e}$, which will result from being shocked first by the moving solid planet and then isentropically being released into space at speeds greater than the escape velocity. This is a conservative calculation since I use the density and pressure of the atmosphere at its base. Moreover, the atmosphere covering the planet closer to the impact than the antipode is expected to achieve high velocity because it is shocked by the decaying air wave, and also shocked to higher pressures by the solid planet. Note that, in general, as a shock wave is propagated upward in an exponential atmosphere, because the density encountered by the traveling shock is decreasing, the shock velocity and particle velocity increases with altitude as discussed in Sect. 6 and by Zel'dovich \& Raizer [1966 Vol. 2, Chaph XII, section 5.25]. Thus, one can safely neglect shock attenuation in the atmosphere, and assume the particle velocity at the solid planet-atmosphere interface (the independent variable) and calculate the shock pressure induced in the gas by the outward surface of the Earth. The solid Earth therefore acts like a piston with velocity, $u_{\mathrm{fs}}$, pushing on the atmosphere.

The pressure behind the shock wave, $p_{1}$, for different outward rock velocities, $u_{f s}$, can be determined by solving for $\mathrm{p}_{1}$ in the following set of equations (e.g. eqs. 1.78+1.79, Z+R): 


$$
\left|u_{f s}\right|=u_{0}-u_{1}
$$

where

$$
u_{0}=\left\{V_{0}\left[(\gamma-1) p_{0}+(\gamma+1) p_{1}\right] / 2\right\}^{1 / 2}
$$

is the particle velocity of the unshocked gas with respect to the shock wave in the atmosphere and

$$
u_{1}=\left\{\frac{v_{0}\left[(\gamma+1) p_{0}+(\gamma-1) p_{1}\right]^{2}}{2\left[(\gamma-1) p_{0}+(\gamma+1) p_{1}\right]}\right\}^{1 / 2}
$$

where $u_{1}$ is the particle velocity of the shocked atmosphere relative to the shock front. Surface values of $p_{0}$ and $V_{0}=1 / \rho_{\infty}$ for the planetary atmospheres used to calculate $p_{1}$ are given in Table 2. Upon isentropic release from $\mathrm{p}_{1}$ to zero pressure, an additional large increase in particle velocity occurs which is given by the Riemann integral:

$$
u_{r}=\int_{0}^{p_{1}}(-d V / d p)^{1 / 2} d p
$$

Upon substituting for an ideal polytropic gas, with a polytropic exponent, $\gamma$, Eq. (20) yields

$$
u_{\mathrm{r}}=\left(\mathrm{p}_{0}^{1 / \gamma} \mathrm{v}_{\mathrm{o}} / \gamma\right)^{1 / 2} \mathrm{p}_{1}^{1 / 2-1 /(2 \gamma) /[1 / 2-1 /(2 \gamma)]}
$$

The shock-induced outward atmospheric velocity, $u_{e}$, is given by:

$$
u_{e}=u_{f s} l+u_{r}
$$

We assume that when

$$
\left|u_{e} \geq v_{e}\right|
$$

atmospheric blow-off occurs. When $v_{e}=v_{e}$, the corresponding value of $u_{f s}$ is denoted by $u_{\text {fse. }}$ The outward rock velocity versus outward atmospheric velocity for the Earth, Venus, 
and Mars, is shown in Fig. 12 for values of the polytropic exponent in the range from 1.1 to 1.3. This range encompasses the effective likely range of $\gamma$ which is expected to decrease from 1.3 to 1.1 with increasing gas ionization. Eq. 23 is satisfied for $\gamma=1.1$ to 1.3 for outward rock velocities of 1.60 to $2.25 \mathrm{~km} / \mathrm{sec}, 1.00$ to $2.45 \mathrm{~km} / \mathrm{sec}$ and 0.27 to $1.2 \mathrm{~km} / \mathrm{sec}$ for the Earth, Venus, and Mars. What impact energies will produce these outward rock velocities for the terrestrial planets?

Fortunately, the strength of the shock-wave induced upon propagation completely through planets with varying iron core sizes overlain by silicate mantles has been recently calculated for objects which have core to planetary radius ratios of 0.333 and 0.466 [Watts et al, 1991]. In these calculations, the energy of the surface source was $E_{w}=3.1 \times 10^{34}$ ergs. Notably, the calculation of Watts et al [1991], when scaled as discussed below, agrees closely with those of Hughes et al [1977] upon which earlier estimates of the energy required to blow-off the Earth's atmosphere were based [Ahrens, 1990].

I scaled the results, which are given as peak compressional wave stresses experienced by material directly beneath the antipode of the impact point for a core to planetary radius ratio of 0.333 and a planet radius, $R_{w}=1500 \mathrm{~km}$, to that for Mars, which has a core to radius ratio of -0.34 and a planetary radius of $R_{p}=3396 \mathrm{~km}$ (Table 2). Similarly, I used the Watts et al. result for a core to planetary radius ratio of 0.47 to provide estimates of the peak stress beneath the antipode for an impact on the Earth and Venus for which the actual core to planetary radius ratios are 0.44 and 0.47 , respectively. Since planetary gravity was not included in the calculations, we employ cube scaling [Melosh, 1989 p. 112] to adjust the results for planetary size.

The energy of the equivalent surface source, $E_{p}$, assumed for an impact on the actual planet of interest is:

$$
E_{p}=E_{w}\left(R_{p} / R_{w}\right)^{3}
$$


To relate the peak pressure experienced by the cell beneath the antipodes for the 0.33 and 0.47 core to planetary radius ratios, a peak shock pressure of $P_{1}=2.03$ and $P_{1}=2.10 \mathrm{GPa}$ were used. To convert these values to shock particle velocity, $u_{1}, I$ assumed a surface density of $p_{0}=2.72 \mathrm{~g} / \mathrm{cm}^{3}$ and a shock velocity $U_{s}=5 \mathrm{~km} / \mathrm{sec}$ in the momentum equation:

$$
u_{1}=P_{1} /\left(\rho_{0} U_{8}\right)
$$

and then made the common approximation that the outward rock (free-surface) velocity $u_{f s w}$ corresponding to the Watts et al. calculation is

$$
u_{\mathrm{fsw}}=2 \mathrm{u}_{1}
$$

The energy, $E_{f s}$ required of an impactor to obtain the upper and lower bounds of $u_{f s}$ necessary to launch the atmosphere to escape velocity in the calculation of Fig. 12, can then be calculated from

$$
E_{f s}=E_{p}\left(u_{f s} / u_{f s w}\right)^{2}
$$

Thus, for complete atmospheric blow-off, values for $E_{\mathrm{fs}}$ of $6.22 \times 10^{37}$ to $1.84 \times 10^{38} \mathrm{ergs}$ are necessary for the Earth. This compares to $-10^{37}$ ergs previously calculated by Ahrens [1990]. For Venus, $E_{f s}$ varies from $2.1 \times 10^{36}$ to $1.3 \times 10^{38}$ ergs, whereas for Mars values of $2.9 \times 10^{35}$ to $5.7 \times 10^{36}$ ergs are needed for complete blow-off (Table 1).

Finally, it is useful to estimate the energy and mass fraction of the planetary atmosphere blown-off in going from the Melosh-Vickery model of tangential blow-off (Table 1) to the condition of complete blow-off. For energies less than those required to eject the entire atmosphere, I assume a simple power law for the decrease of particle velocity with radius, $r$, from the impact point (Fig. 11b) and assume that as the decaying stress wave interacts with the free-surface of a spherical planet, atmospheric blow-off occurs if the particle velocity is greater or equal to $0.5 u_{\mathrm{fse}}$, which is the value of $u_{\mathrm{fs}}$, required to blow off the atmosphere for the antipodal case. Thus, the amount of atmosphere blown-off is related to the area of a sphere subtended by an arc of radius, $r$, at 
the point where the particle velocity has decayed to $0.5 u_{\text {fse }}$ (Fig. 11b).

To determine an empirical relation for the attenuation of particle velocity, I assume the form [Melosh, 1989 p. 62].

$$
\mathbf{u}=\mathrm{u}_{\mathbf{0}} / \mathbf{r}^{\mathbf{n}}
$$

I first calculate the radius of a hemisphere, $r_{1}$, enclosing a unit mass

$$
r_{1}=\left[3 /\left(2 \pi \rho_{0}\right)\right]^{1 / 3}
$$

Using the values of $E_{f s} \min$ and $E_{f s} \max$ calculated from eq. 27 to designate the minimum and maximum energies obtained from Fig. 12 for $\gamma=1.1$ and $\gamma=1.3$, respectively, the shock particle velocity associated with each energy is given by

$$
\begin{aligned}
& u_{1 \min }=\left(E_{f s \min }\right)^{1 / 2} \\
& u_{1 \max }=\left(E_{f s \max }\right)^{1 / 2}
\end{aligned}
$$

Denoting $l_{\mathrm{fs}} \mid$ calculated from Eq. 17 using $\gamma=1.1$ and $\gamma=1.3$ as $u_{f s} \min$ and $u_{f s} \max I$ can obtain from Eqs. 26 and 28, expressions for the particle velocity decay parameters $n_{1}$ and $\mathbf{n}_{2}$ from

$$
\begin{aligned}
& \mathrm{n}_{1}=\log \left(2 u_{1 \min } / u_{f s \min }\right) / \log \left(2 R_{p} / r_{1}\right) \\
& n_{2}=\log \left(2 u_{1 \max } / u_{f_{s} \max }\right) / \log \left(2 R_{p} / r_{1}\right)
\end{aligned}
$$

For stress wave particle velocity decaying to a value of $u_{f s w} / 2$ at a radius from the impact point $r_{e}$, the mass of atmosphere blown off is

$$
m_{e}=\left(p_{d} / g\right) A
$$

where the term in parenthesis is the atmospheric mass per unit area and $A$ is the area of a sphere subtended by an arc of length, r, (Fig. 11b).

From geometrical arguments it can be shown that the area of the planet subtended by an arc of length, $r$, is

$$
A=\pi r^{2}
$$

The energies (minimum and maximum) associated with the radius, $r_{e}$, for $\gamma=1.3$ and $\gamma=$ 
1.1 values are:

$$
\begin{aligned}
& E_{\min }=\left[u_{f s \min }\left(r_{d} / r_{1}\right)^{n_{1} / 2}\right]^{2} \\
& E_{\max }=\left[u_{f s \max }\left(r_{d} / r_{1}\right)^{n_{1} / 2}\right]^{2}
\end{aligned}
$$

The normalized mass of the atmosphere blown-off, $m_{d} / m_{a}$ versus both $E_{\min }$ and $E_{\max }$ are shown in Fig. 13.

Table 1 shows that the energy for atmospheric loss above a tangent plane is a small fraction $\left(-10^{-8}\right.$ to $\left.10^{-4}\right)$ of the energy required to drive-off the entire atmosphere. For the Earth, this total loss energy is $\sim 10^{38}$ ergs and would be achieved via an impact of a lunarsized object at $20 \mathrm{~km} / \mathrm{sec}$. In the case of Venus, the impact of a smaller $-800 \mathrm{~km}$ radius object, at $-20 \mathrm{~km} / \mathrm{sec}$, will drive off the atmosphere. For Mars, the impact of a $160 \mathrm{~km}$ radius object at $20 \mathrm{~km} / \mathrm{sec}$ will drive off the atmosphere. It may be, as suggested by Cameron [1983], that the terrestrial planets all suffered several giant impacts and their present atmospheres may reflect accretion and outgassing since the last great impact event.

\section{ACKNOWLEDGMENTS}

I appreciate receiving private communications from G. Chen, A. Vickery, and J. Melosh. Many of the ideas developed in this review have come from collaborations with J. D. O'Keefe, M. A. Lange, and J. A. Tyburczy. I appreciate helpful comments on this paper proffered by A. W. Harris and L. R. Rowan. Research supported by NASA. Contribution \#5198, Division of Geological and Planetary Sciences, California Institute of Technology. 


\section{FIGURE CAPTIONS}

Figure 1. Formation of asteroid-size intermediate bodies form the dust component of the solar nebula a conditional variant assuming (a) instantaneous formation of the solar nebula and (b) breakup of the dust disc caused by the onset of gravitational instability. There is gradual accretion of intermediate bodies into planets. The accretion of gas by giant planets is not shown. The initially flat system of intermediate bodies thickens due to their mutual gravitational perturbations. (after Levin, [1972]).

Figure 2. Enhanced image of the star $\beta$-Pictoris demonstrates what appears to be the beginnings of another solar system. The disk of material surrounding $\beta$-Pictoris extends $60 \times 10^{9} \mathrm{~km}$ from the star, which is located behind a circular occulting mask in the center of the image. The disc material is probably composed of gases and grains of ices, carbonaceous chondrite-like organic substances, and silicates. These are the materials from which the comets, asteroids, and planets of our own solar system are thought to have formed (after Smith \& Terrile [1984]).

Figure 3. Atomic abundance of the elements in the solar photosphere vs. the abundance in chondritic meteorites. Plot is normalized with respect to $10^{6}$ atoms of Si (after Allègre, [1982]).

Figure 4. Abundances of noble gases in planetary atmospheres and chondritic meteorites given as cubic centimeters per gram of rock (after E. Anders, personal communication in Owen et al [1992]). 
Figure 5. Cartoon indicating the difference between the thermal regime of accretion via "thermal blanketing" and previous studies (figure from Abe \& Matsui [1985]).

Figure 6. (a) The evolution of surface temperature during accretion of a model Earth from planetesimals containing $0.1 \% \mathrm{H}_{2} \mathrm{O}$. The radius, $\mathrm{R}$, is normalized by the final value, $\mathbf{R}_{\mathbf{0}}$. The dashed curve gives the calculated surface temperature without an impact-generated atmosphere (accretion period is $5 \times 10^{7} \mathrm{yr}$ ). The model surface temperature is affected by an atmosphere (which begins to greatly increase its mass once the impact velocity exceeds a critical value). The rapid rise in the surface temperature of the "standard model" which occurs after the Earth grows to $-0.3 R_{0}$, is due to an increase in the total mass of the atmosphere because of the initiation of the complete dehydration reaction of the surface layer. Once the surface temperature reaches the melting temperature, it remains nearly constant (figure after Matsui \& Abe [1986a]). (b) The total mass of the impact-generated $\mathrm{H}_{2} \mathrm{O}$ atmosphere is plotted against the normalized radius for the standard planetesimal models. Note that the total atmospheric mass, $\mathbf{M}_{\mathbf{S}}$, remains nearly constant after the Earth grows to $0.4 \mathrm{R}_{\mathrm{O}}$ and is very close to the present mass of the Earth's oceans $\left(1.4 \times 10^{21} \mathrm{~kg}\right.$ ) (figure after Matsui \& Abe [1986a]).

Fig. 7. Shock front at successive instants of time for a strong explosion at high altitude. Sections shown are formed by passing a vertical plane through the origin of the explosion. The density of the atmosphere changes by a factor of $e$ over the atmospheric scale height, $\Delta$. Note that $\tau=\left(\rho_{c} \Delta^{5 / E}\right)^{1 / 2}$ where $\rho_{c}$ is the density at the altitude of the explosion and $\mathrm{E}$ is the energy (after Zel'dovich \& Raizer [1966]).

Fig. 8. Cartoons of atmospheric impact indicating how projectile momentum can be 
transferred to a planetary atmosphere. A) The projectile enters the atmosphere, heating, compressing, and accelerating the atmospheric gases ahead of it. B) Solid ejecta from a growing crater pass through the atmosphere, transferring some or all of their momentum to the atmosphere by drag. Only a small quantity of atmosphere is ejected via the mechanisms of A and B. C) The impact-generated vapor plume expands upward and outward (after Vickery \& Melosh [1990]).

Fig. 9. The impact-generated gas is assumed to interact only with the atmosphere lying above the plane tangent to the earth at the center of impact (after Vickery \& Melosh [1990]).

Fig. 10. The normalized maximum atmospheric mass that can be expelled by an impacting veneer of normalized mass for three values of $q$, the power law exponent describing the (differential) mass spectrum of impactors. Mars (dots) and Venus (dashes) are quantitatively similar (both shown for $q=1.7$ ). Rectangles indicate veneer masses (width corresponds to plausible $\mathrm{q}$ values) needed to remove present atmospheres of Titan, Mars, and Venus (after Zahnle et al [1988]).

Fig. 11. (a) Sketch of lunar-sized planetesimal impacting the Earth. The protoatmosphere is blown away by the shock wave-induced motion of the solid or molten planet. (b) Impact of asteroidal-sized impactor and resultant partial eroded atmosphere.

Fig. 12. Relationship of outward free-surface velocity $\left(u_{\mathrm{fs}}\right)$ to outward atmospheric velocity $\left(v_{e}\right)$ for a polytropic atmosphere with various values of $\gamma$. (a) Earth, (b) Venus, (c) Mars.

Fig. 13. Calculated fraction of atmosphere blown-off versus impactor energy for Earth, Venus, and Mars. Lower and higher energy curves for each planet corresponds to assumed polytropic exponent of ideal gas of $\gamma=1.1$ and 1.3, respectively. 


\section{REFERENCES}

Abe, Y., Matsui, T. 1985. The formation of an impact-generated $\mathrm{H}_{2} \mathrm{O}$ atmosphere and its implications for the early thermal history of the Earth. Proc. Lunar Planet. Sci. Conf. 15th, Part 2, J. Geophys. Res. 90:C545-C559.

Abe, Y., Matsui, T. 1986. Early evolution of the Earth: Accretion, atmosphere formation and thermal history. Proc. XVII Lunar and Planet. Sci. Conf., I, J. Geophys. Res. 91:E291-E302.

Abe, Y., Matsui, T. 1988. Evolution of an impact-generated H20-C02 atmosphere and formation of a hot proto-ocean on earth. J. Atmos. Science 45:3081-3101.

Ahrens, T. J. 1990. Earth Accretion. In Origin of the Earth, ed. J. Jones, H. Newsom, 211-227. Houston, TX: Oxford U. Press.

Ahrens, T. J., O'Keefe, J. D. 1987. Impact on the Earth, ocean, and atmosphere. Proc. Hypervelocity Impact Symp., Int. J. Impact Engng. 5:13-32.

Ahrens, T. J., O’Keefe, J. D., Lange, M. A. 1989. Formation of atmospheres during accretion of the terrestrial planets. In Origin and Evolution of Planetary and Satellite Atmospheres, ed. S. K. Atreya, J. B. Pollack, M. S. Matthews, 328-385. Tucson, AZ: University of Arizona Press.

Allègre, C. J. 1982. Cosmochemistry and primitive evolution of planets. In Formation of Planetary Systems, ed. A. Brahic, 283-364. Paris: Centre National d'Etudes 
Spatiales.

Arrhenius, G., De, B. R., Alfven, H. 1974. Origin of the ocean. In The Sea, ed. E. D. Goldberg, 839-861. New York: Wiley.

Bach, G. G., Kuhl, A. L., Oppenheim, A. K. 1975. On blast waves in exponential atmospheres. J. Fluid Mech. 71:105-122.

Beckwith, S., Sargent, A. I., Scoville, N. Z., Masson, C. R., Zuckerman, B., et al. 1986. Small-scale structure of the circumstellar gas of H. L. Tauri and R. Monocerotis. Astrophys. J. 309:755-761.

Benlow, A., Meadows, A. J. 1977. The formation of the atmospheres of the terrestrial planets by impact. Astrophys. Space Sci. 46:293-300.

Benz, W., Cameron, A. G. W., Melosh, H. J. 1989. The origin of the moon and the single impact hypothesis, III. Icarus 81:113-131.

Benz, W., Slattery, W. L., Cameron, A. G. W. 1986. The origin of the moon and the single impact hypothesis, I. Icarus 66:515-535.

Benz, W., Slattery, W. L., Cameron, A. G. W. 1987. Origin of the moon and the single impact hypothesis, II. Icarus :30-45.

Benz, W., Slattery, W. L., Cameron, A. G. W. 1988. Collisional stripping of Mercury 
mantle. Icarus 74:516-528.

Cameron, A. G. W. 1983. Origin of the atmospheres of the terrestrial planets. Icarus 56:195-201.

Donahue, J. M., Hodges, R. R., Jr. 1992. Past and present water budget of Venus. J. Geophys. Res. 97:6083-6092.

Goldreich, P., Ward, W. R. 1973. The formation of planetesimals. Astrophys. J. 183:1051-1061.

Greenberg, R. 1989. Planetary accretion. In Origin and Evolution of Planetary and Satellite Atmospheres, ed. S. K. Atreya, J. B. Pollack, M. S. Matthews, 137164. U. Ariz. Press.

Grinspoon, D. H., Lewis, J. S. 1988. Cometary water on Venus: Implications for stochastic impacts. Icarus 74:21-35.

Hughes, H. G., App, F. N., McGetchen, T. R. 1977. Global effects of basin-forming impacts. Phys. Earth and Planet. Int. 15:251-263.

Hunten, D. M., Pepin, R. O., Owen, T. C. 1989. Planetary atmospheres. In Meteorites and the Early Solar System, ed. J. F. Kerridge, M. S. Matthews, 565-594. Univ. Ariz. Press. 
Jessberger, E. K., Kissel, J., Rahe, J. 1989. The composition of comets. In Origin and Evolution of Planetary Satellite Atmospheres, ed. S. K. Atreya, J. B. Pollack, M. S. Matthews, 167-191. U. Ariz. Press.

Jones, E. M., Kodis, J. W. 1982. Atmospheric effects of large body impacts; The first few minutes. In Geological Implications of Impacts of Large Asteroids and Comets on the Earth, ed. L. T. Silver, P. H. Schultz, 175-186. Geol. Soc. Amer. Special Paper.

Kaula, W. M. 1979. Thermal evolution of Earth and Moon growing by planetesimal impacts. J. Geophys. Res. 84:999-1008.

Kipp, M. E., Melosh, H. J. 1986. Short note: A preliminary study of colliding planets. In Origin of the Moon, ed. W. K. Hartmann, R. J. Phillips, G. T. Taylor, 643648. Lunar and Planet. Inst.

Lange, M. A., Ahrens, T. J. 1982a. The evolution of an impact-generated atmosphere. Icarus 51:96-120.

Lange, M. A., Ahrens, T. J. 1982b. Impact-induced dehydration of serpentine and the evolution of planetary atmospheres. Proc. Lunar Planet. Sci. Conf. 13th, Part 1, J. Geophys. Res., Supplement 87:A451-A456.

Levin, B. J. 1972. Origin of the earth. In The Upper Mantle, ed. A. R. Ritsema, 7-30. Elsevier. 
Matsui, T., Abe, Y. 1986a Evolution of an impact-induced atmosphere and magma ocean on the accreting Earth. Nature 319:303-305.

Matsui, T., Abe, Y. 1986b. Impact induced atmospheres and oceans on Earth and Venus. Nature 322:526-528.

Melosh, H. J., 1989. Impact Cratering, A Geologic Process. New York. Oxford University Press. 245 pp.

Melosh, H. J., Vickery, A. M. 1989. Impact erosion of the primordial Martian atmosphere. Nature 338:487-489.

O'Keefe, J. D., Ahrens, T. J. 1977a. Impact induced energy partitioning, melting, and vaporization on terrestrial planets. Proc. Lunar Sci. Conf 8 th :3357-3374.

O'Keefe, J. D., Ahrens, T. J. 1977b. Meteorite impact ejecta: Dependence on mass and energy lost on planetary escape velocity. Science 198:1249-1251.

O'Keefe, J. D., Ahrens, T. J. 1982. The interaction of the Cretaceous-Tertiary extinction bolide with the atmosphere, ocean, and solid earth. Geol. Soc. Am. Spec. Paper, $190,103-120$.

Owen, T., Bar-Nunn, A., Kleinfeld, I. 1992. Possible cometary origin of heavy noble gases in the atmospheres of Venus, Earth, and Mars. Nature 358:43-45. 
Pollack, J. B., Bodenheimer, P. 1989. Theories of the origin and evolution of the giant planets. In Origin and Evolution of Planetary and Satellite Atmospheres, ed. S. K. Atreya, J. B. Pollack, M. S. Matthews, 564-602. U. Ariz. Press.

Ringwood, A. E., 1979. Origin of the Earth and Moon. Berlin, New York. SpringerVerlag. 295 pp.

Safronov, V. S. 1969. Evolution of the Proto-Planetary Cloud and Formation of the Earth and Planets, Moscow: Nauka. Transl. for NASA and NSF by Isr. Program Sci. Transl. as NASA-TT-F677.

Sargent, A. I., Beckwith, S. 1987. Kinematics of the circumstellar gas of HL Tauri and R Monocerotis. Astrophys. J. 323:294-305.

Sargent, A. I., Beckwith, S. V. 1992. The molecular structure around HL Tauri. Astrophys. J. (Letters) :in press.

Shoemaker, W. M., Wolfe, R. F., Shoemaker, C. S. 1990. Asteroid and comet flux in the neighborhood of Earth. In Global Catastrophes in Earth History, ed. V. L. Sharpton, P. D. Ward, 155-170. Geol. Soc. Am. Sp. Paper.

Smith, B. A., Terrile, R. J. 1984. A circumstellar disk around ß-Pictoris. Science 226:1421-1424. 
Vickery, A. M., Melosh, H. J. 1990. Atmospheric erosion and impactor retention in large impacts with application to mass extinctions. In Global Catastrophes in Earth History, ed. V. L. Sharpton, P. O. Ward, 289-300. Geol. Soc. Am. Sp. Paper.

Walker, J. C. G. 1986. Impact erosion of planetary atmospheres. Icarus 68:87-98.

Watts, A. W., Greeley, R., Melosh, H. J. 1991. The formation of terrains antipodal to major impacts. Icarus 93:159-168.

Wetherill, G. W. 1980. Formation of the terrestrial planets. Ann. Rev. Astron. Astrophys. 18:77-113.

Wetherill, G. W. 1985. Occurrence of giant impacts during the growth of the terrestrial planets. Science 228:877-879.

Wetherill, G. W., Stewart, G. R. 1989. Accumulation of a swarm of small planetesimals. Icarus 77:330-357.

Zahnle, K. J., Kasting, J. F., Pollack, J. B. 1988. Evolution of a steam atmosphere during Earth's accretion. Icarus 74:62-97.

Zel'dovich, Y. B., Raizer, Y. P., 1966. Physics of Shock Waves and High-Temperature Hydrodynamic Phenomena. Vol. 112. New York. Academic Press. 916 pp. 

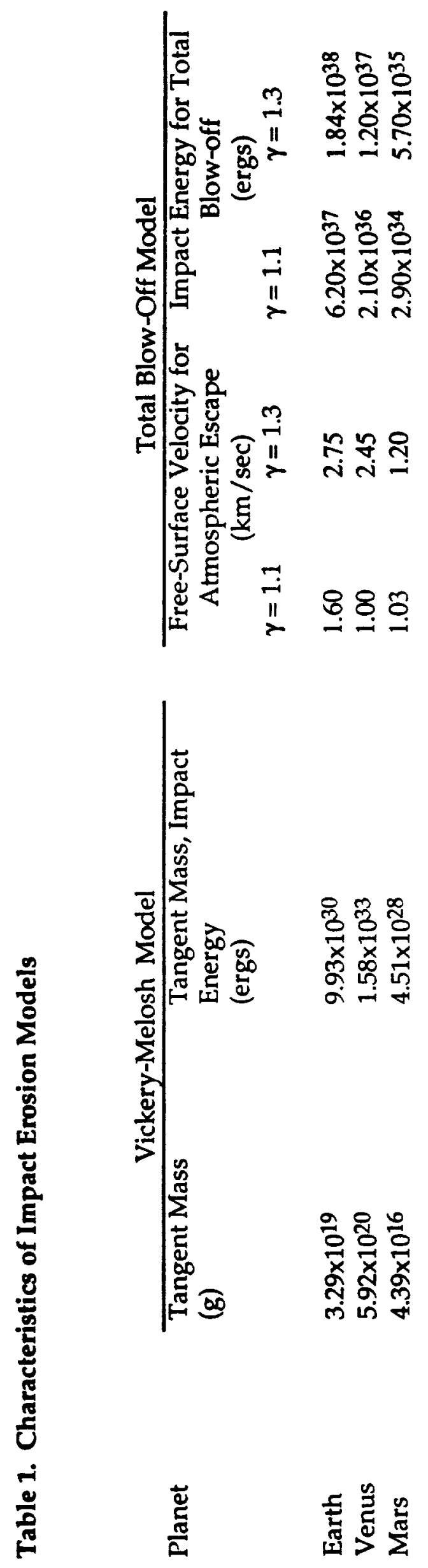


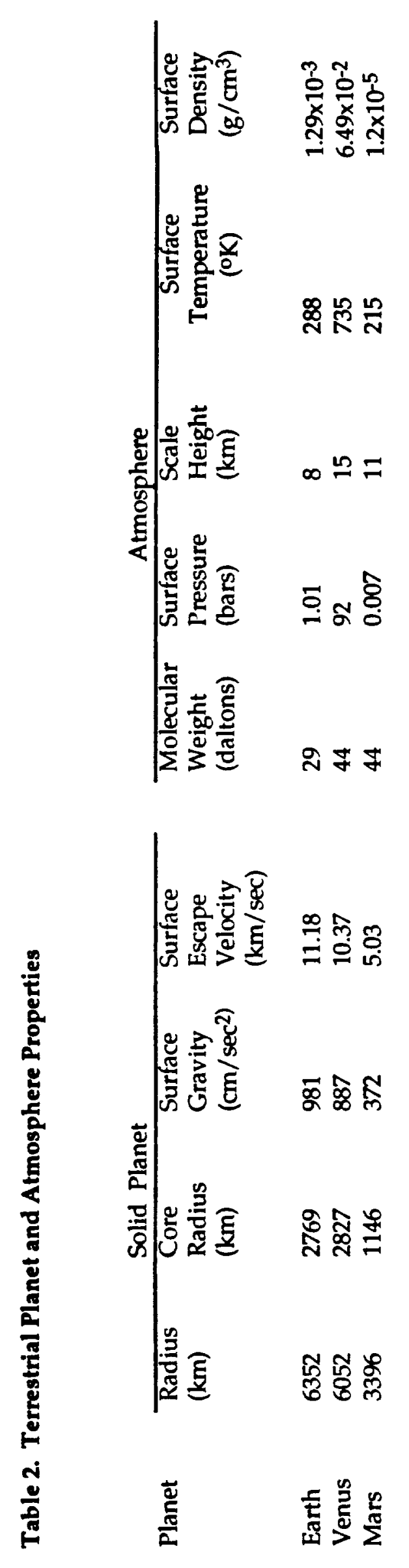




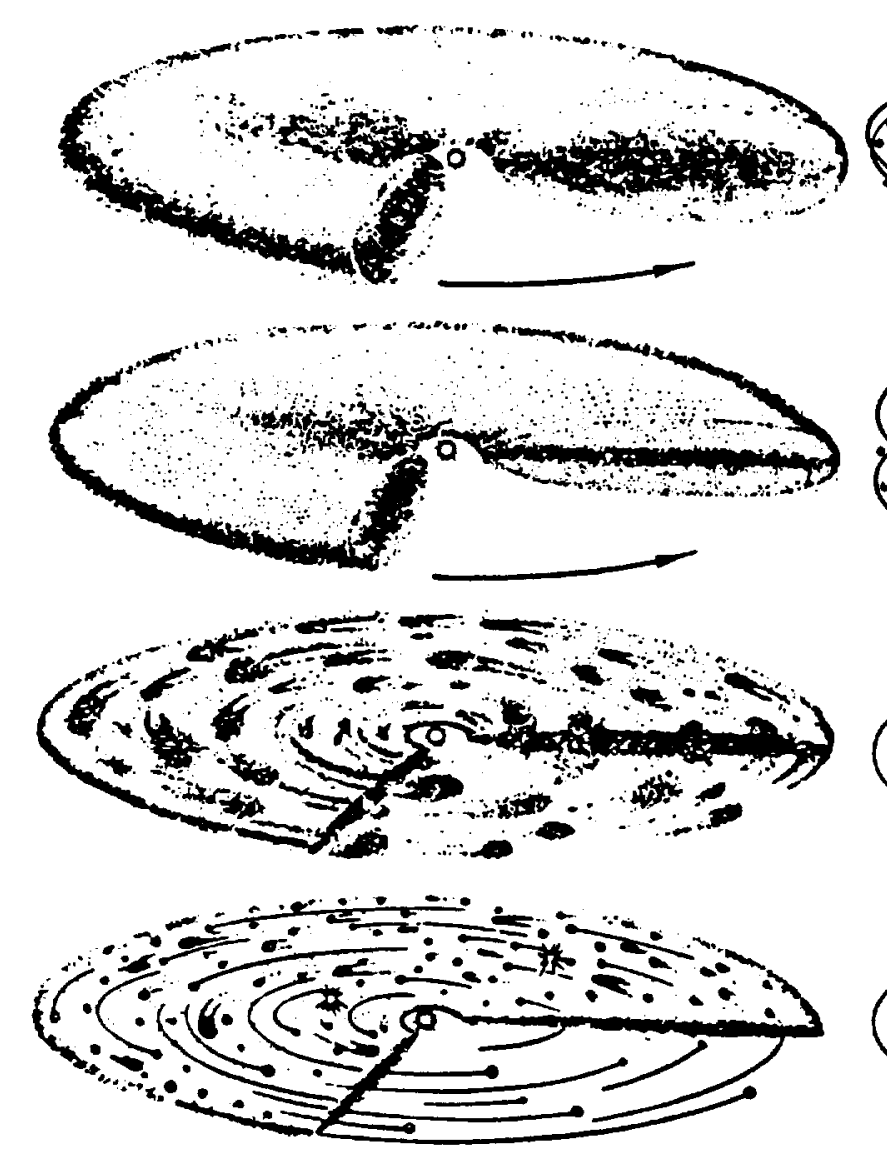

a
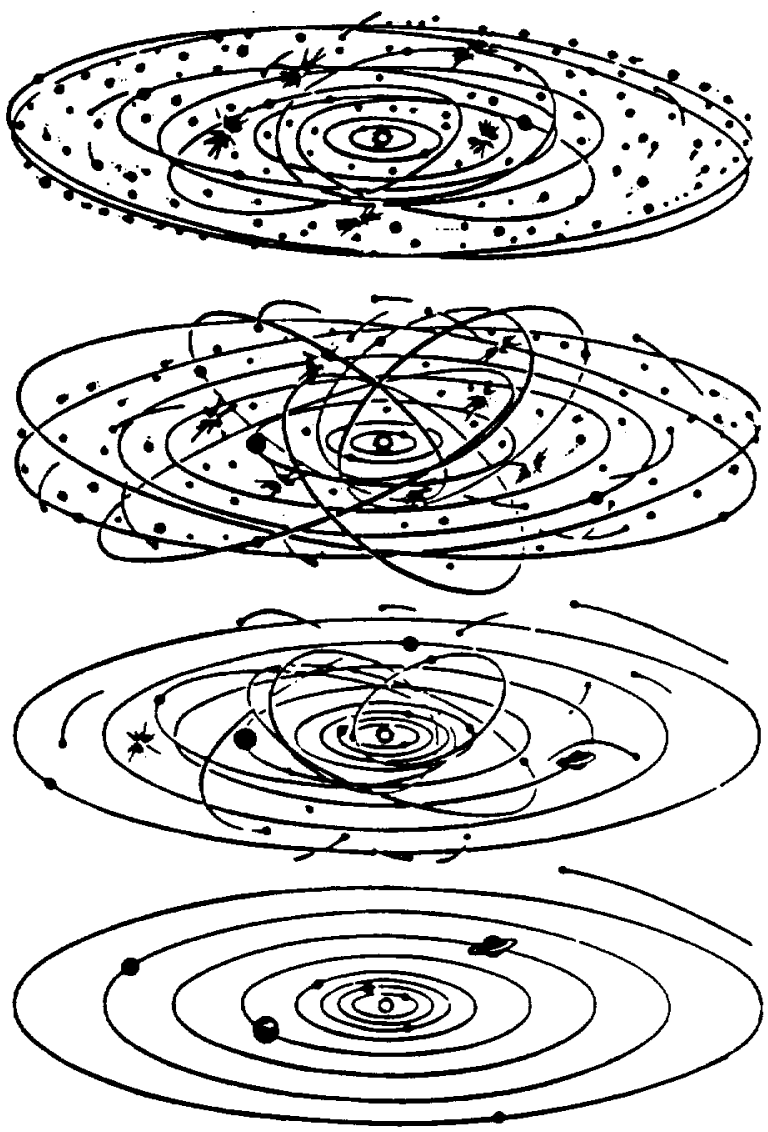

b 
告 Ap

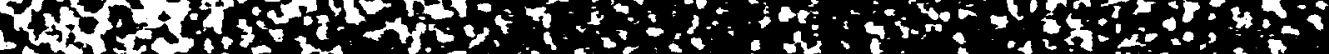

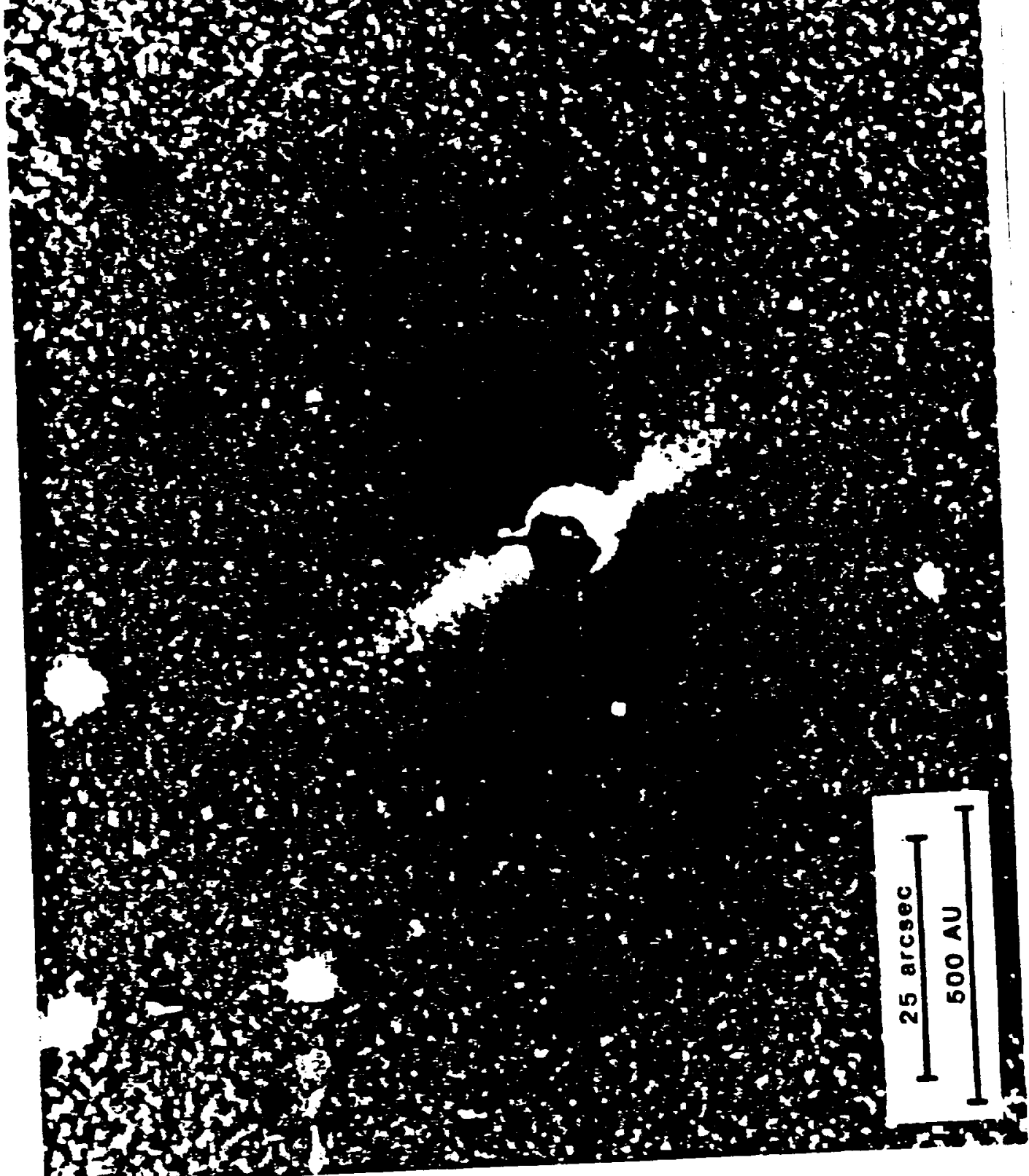




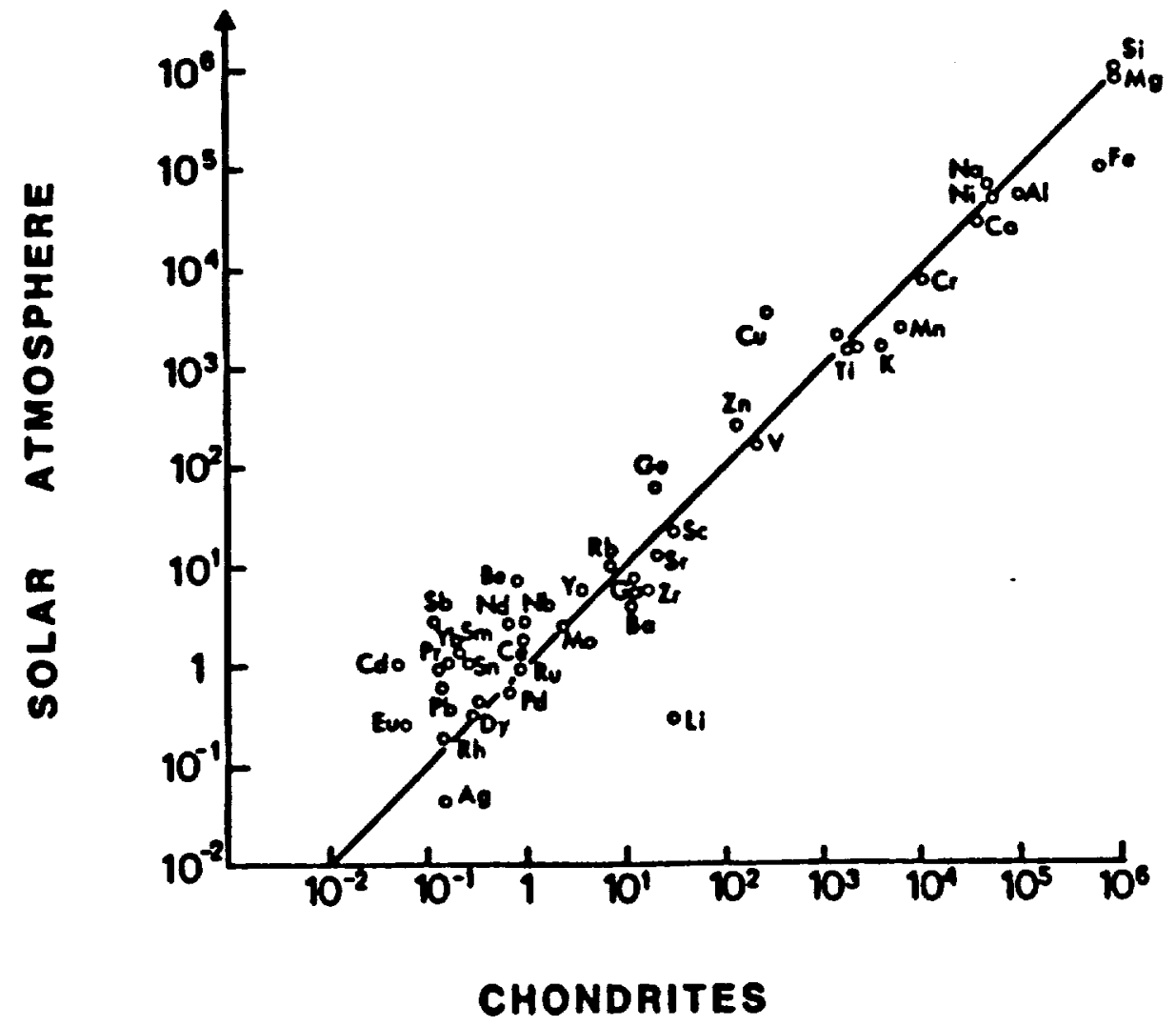

Fig. 3 


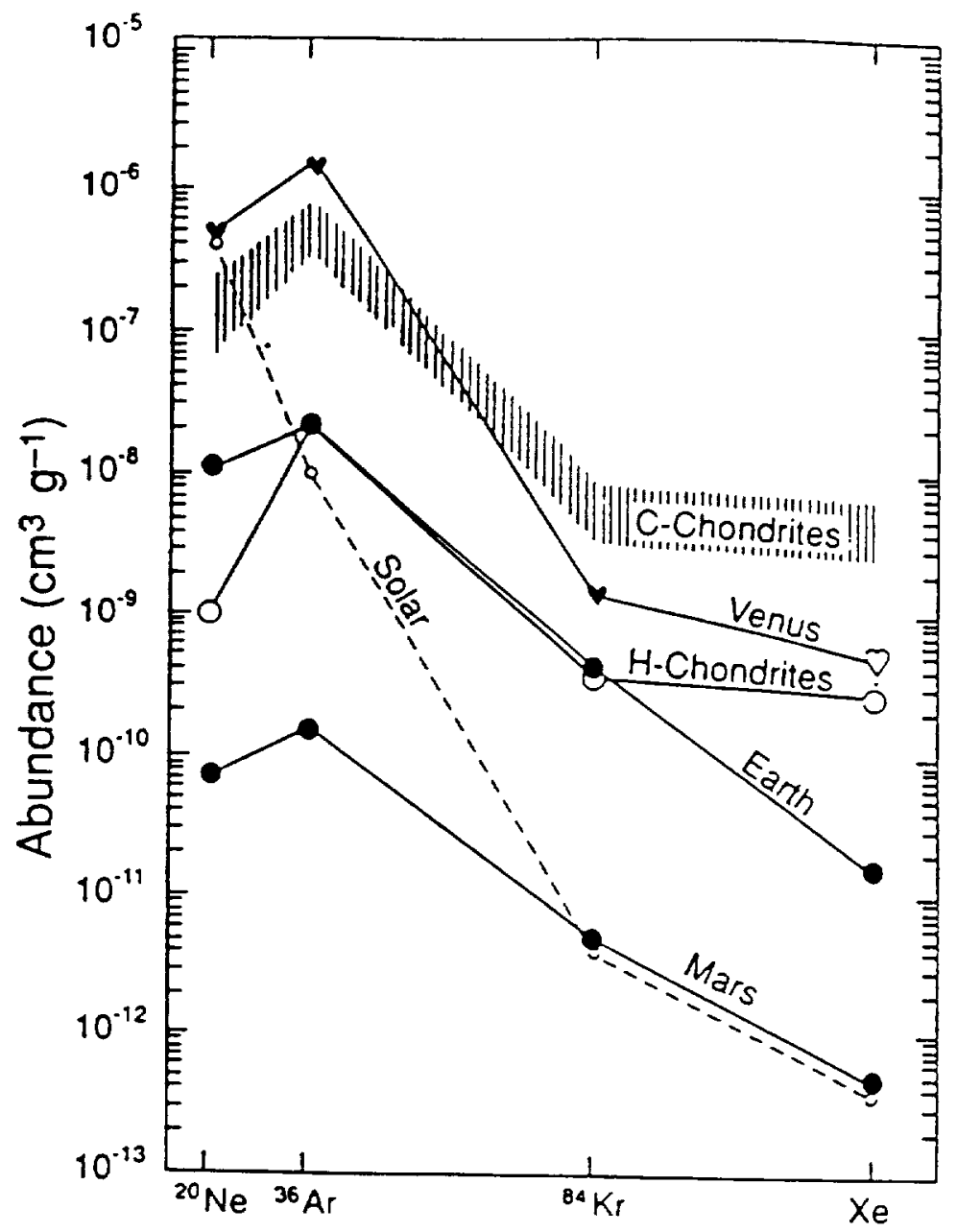



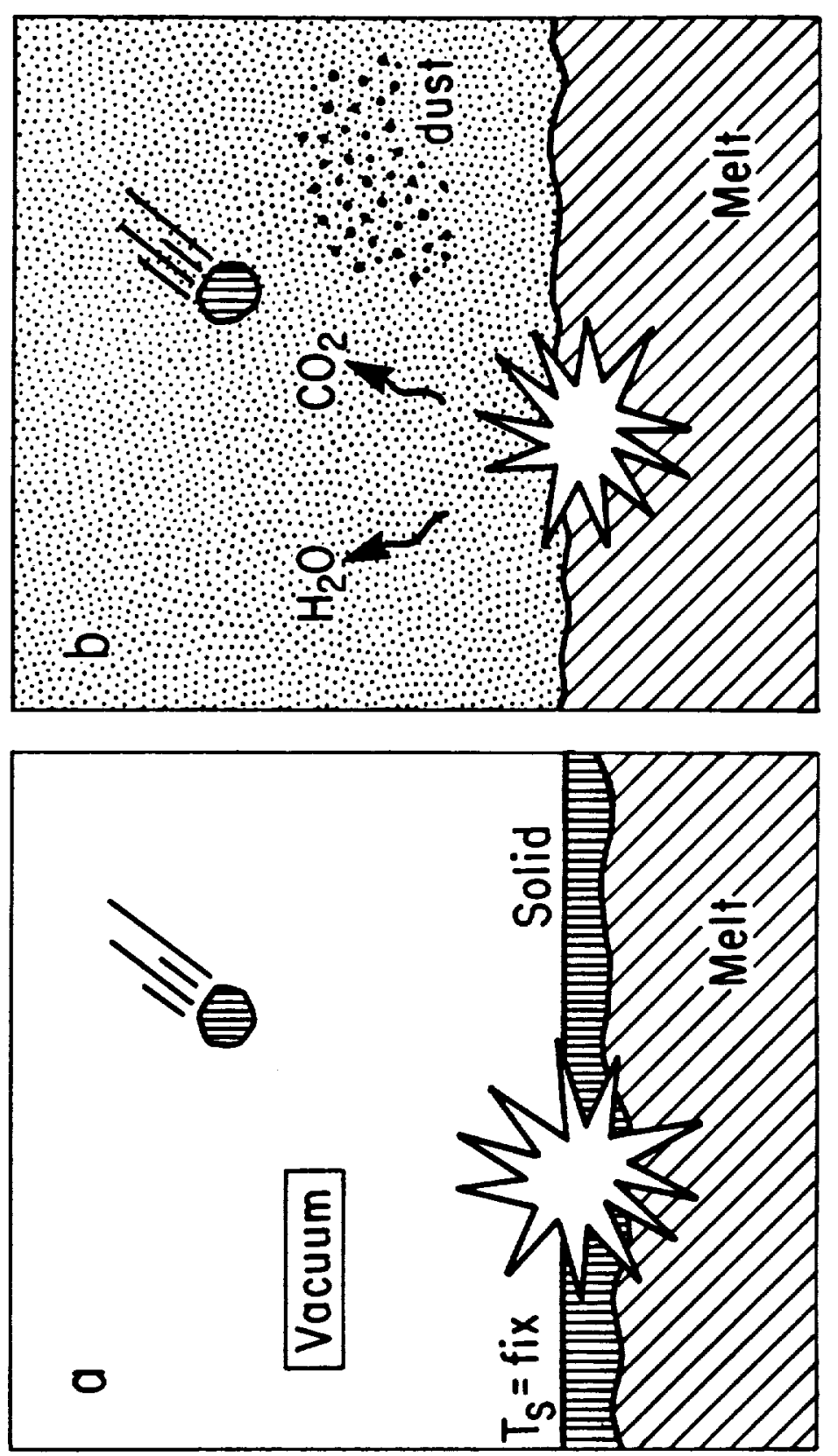


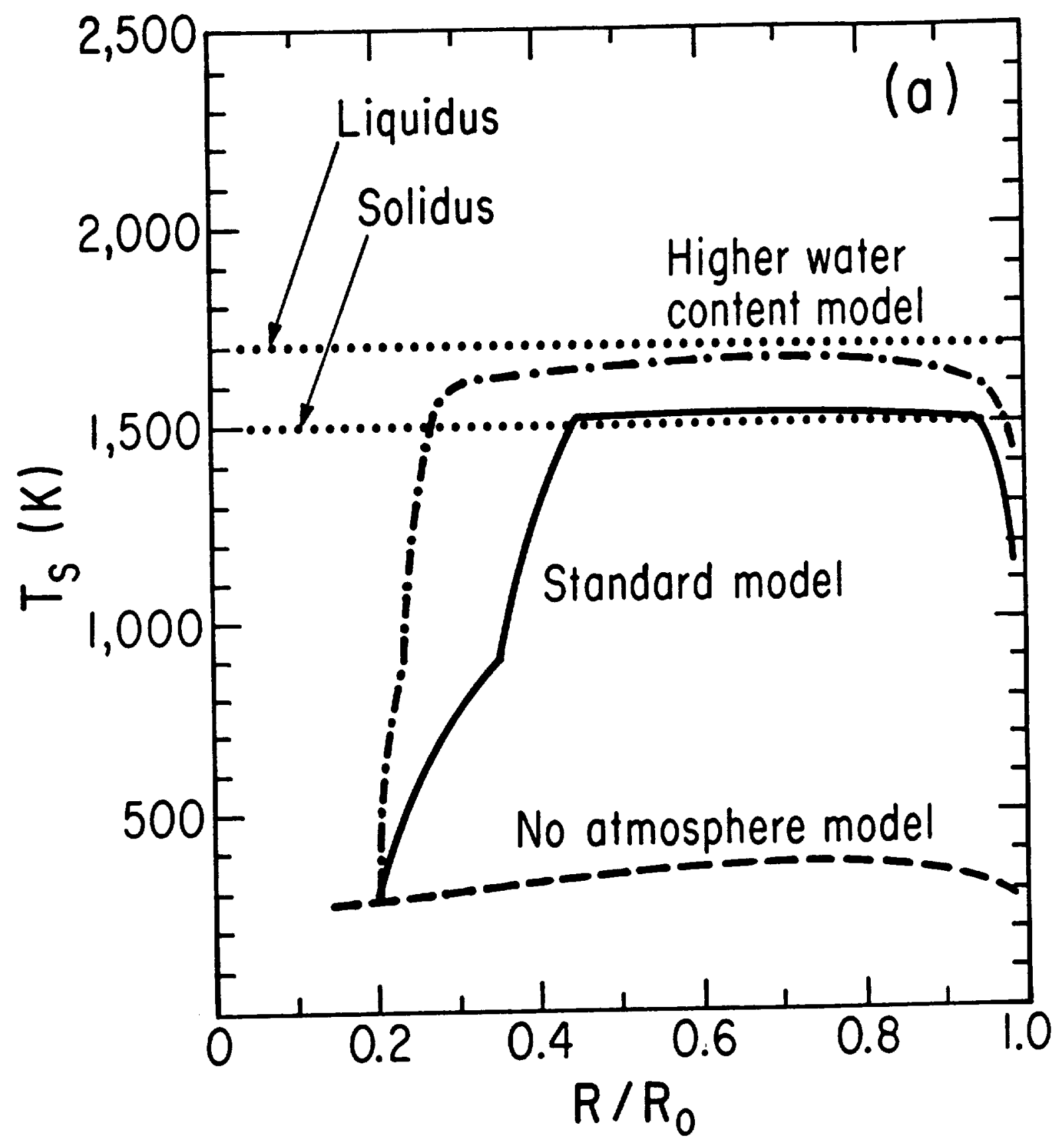




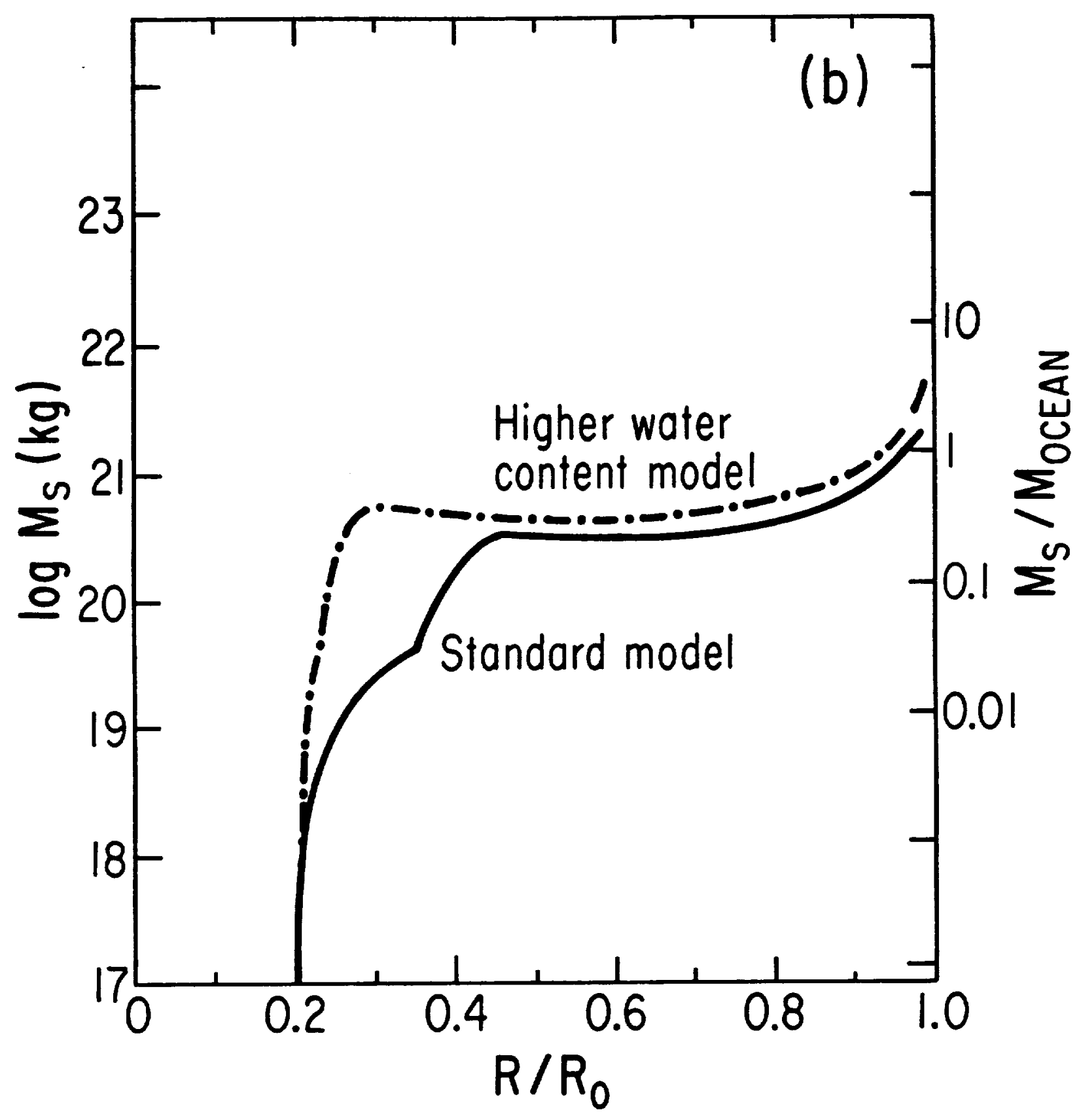

TJA87155SFD 


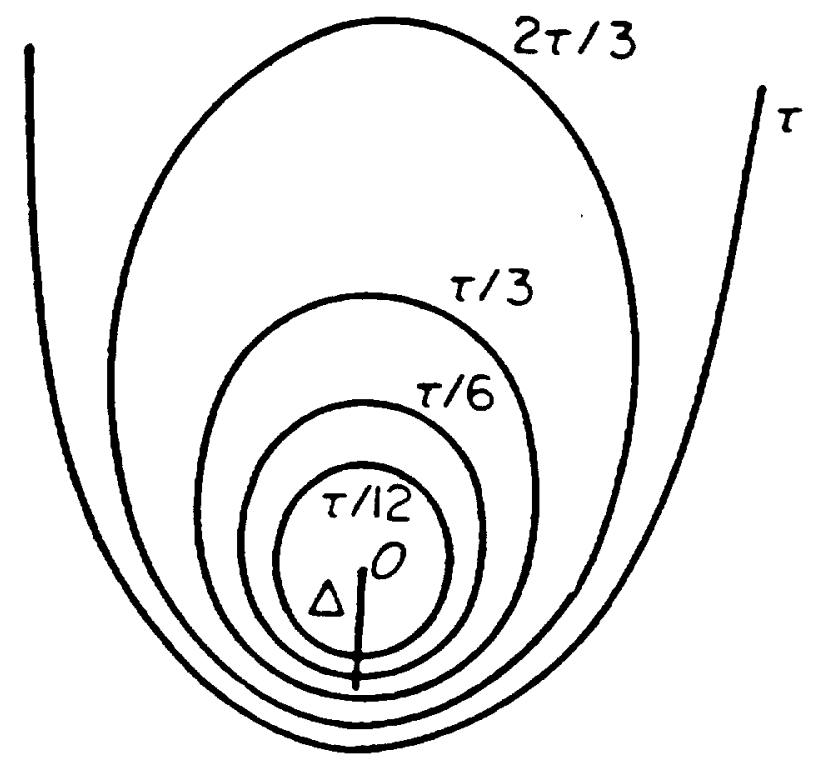



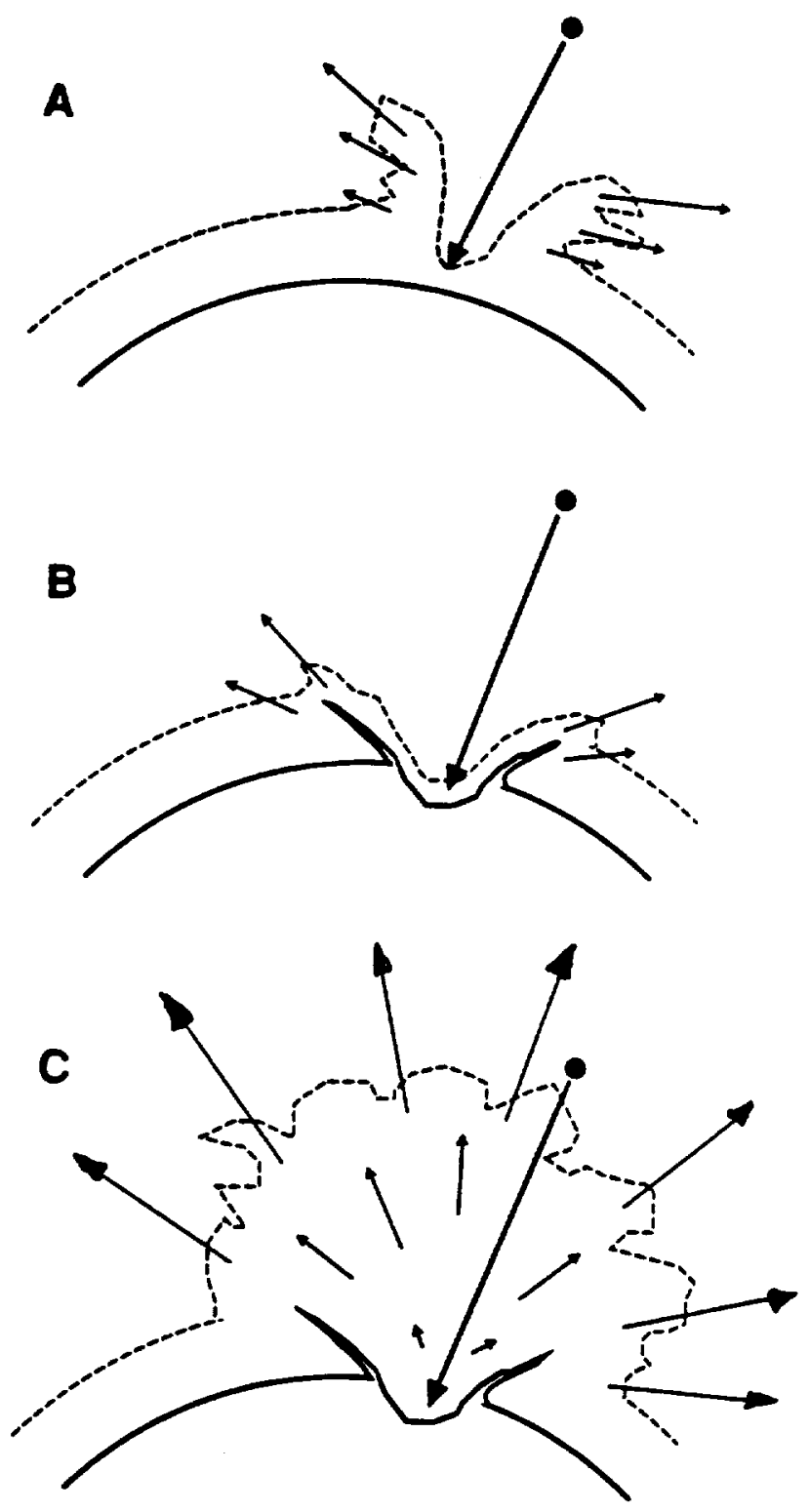


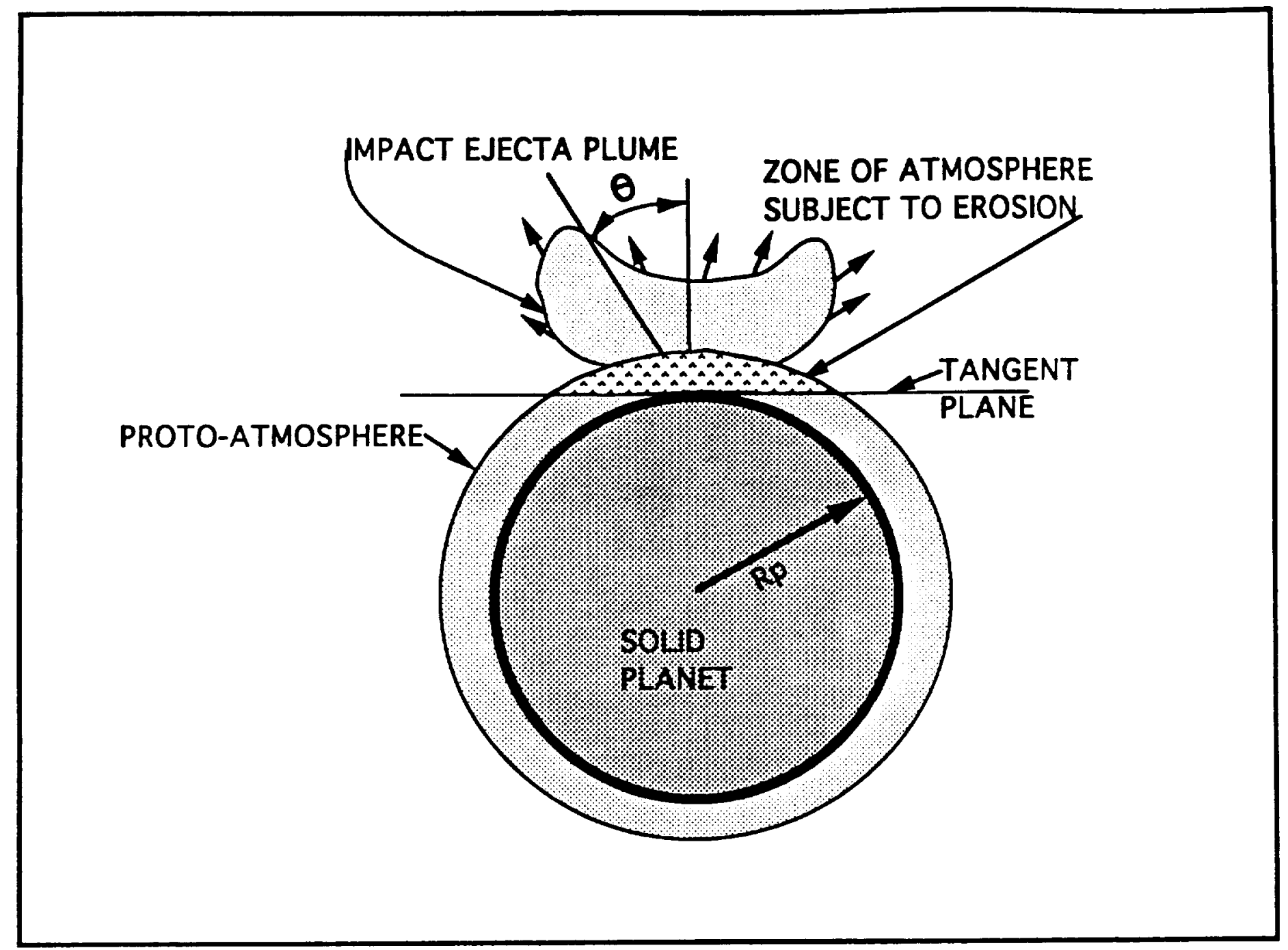




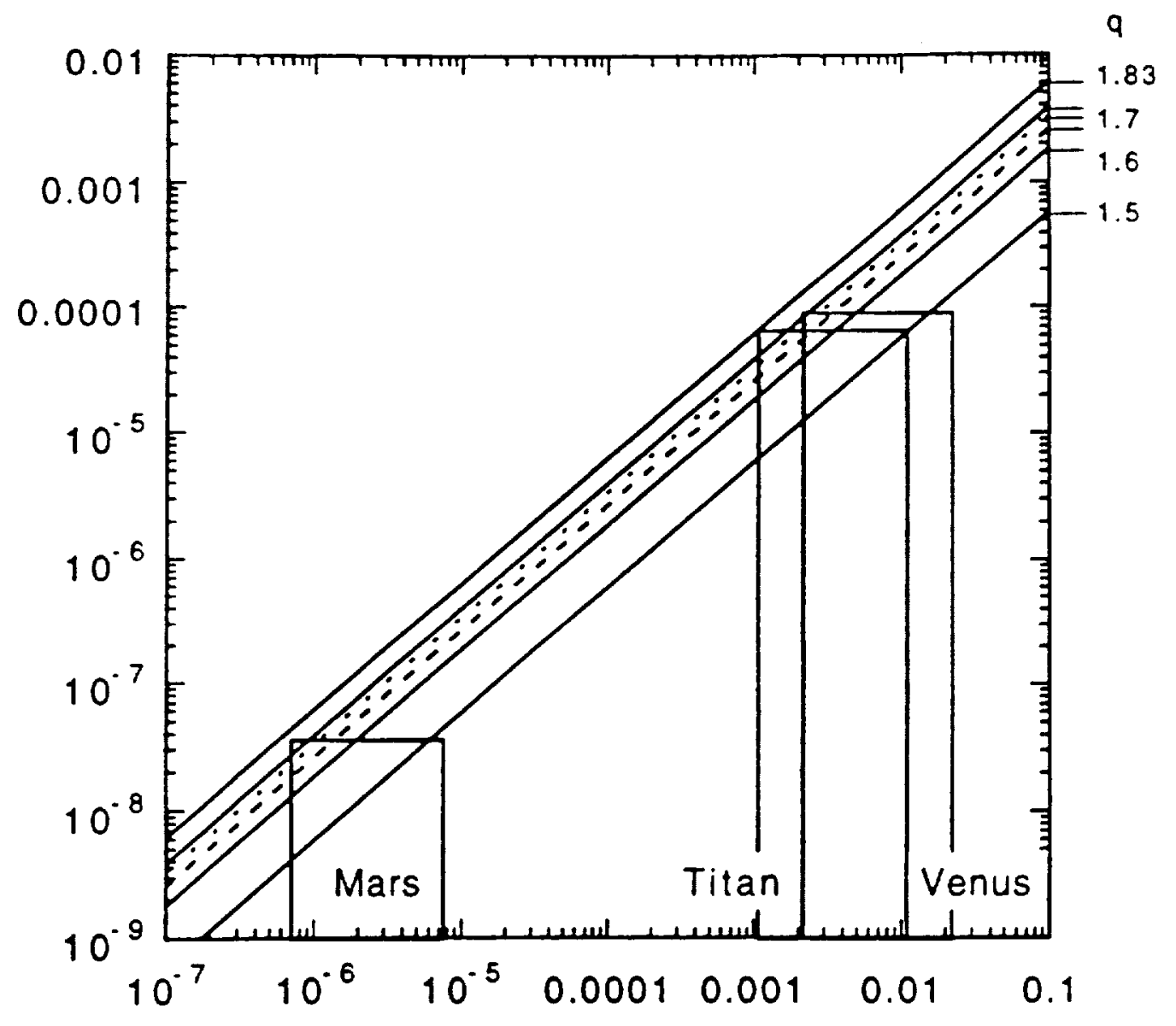




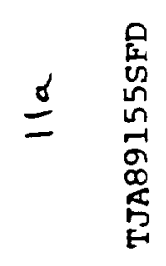

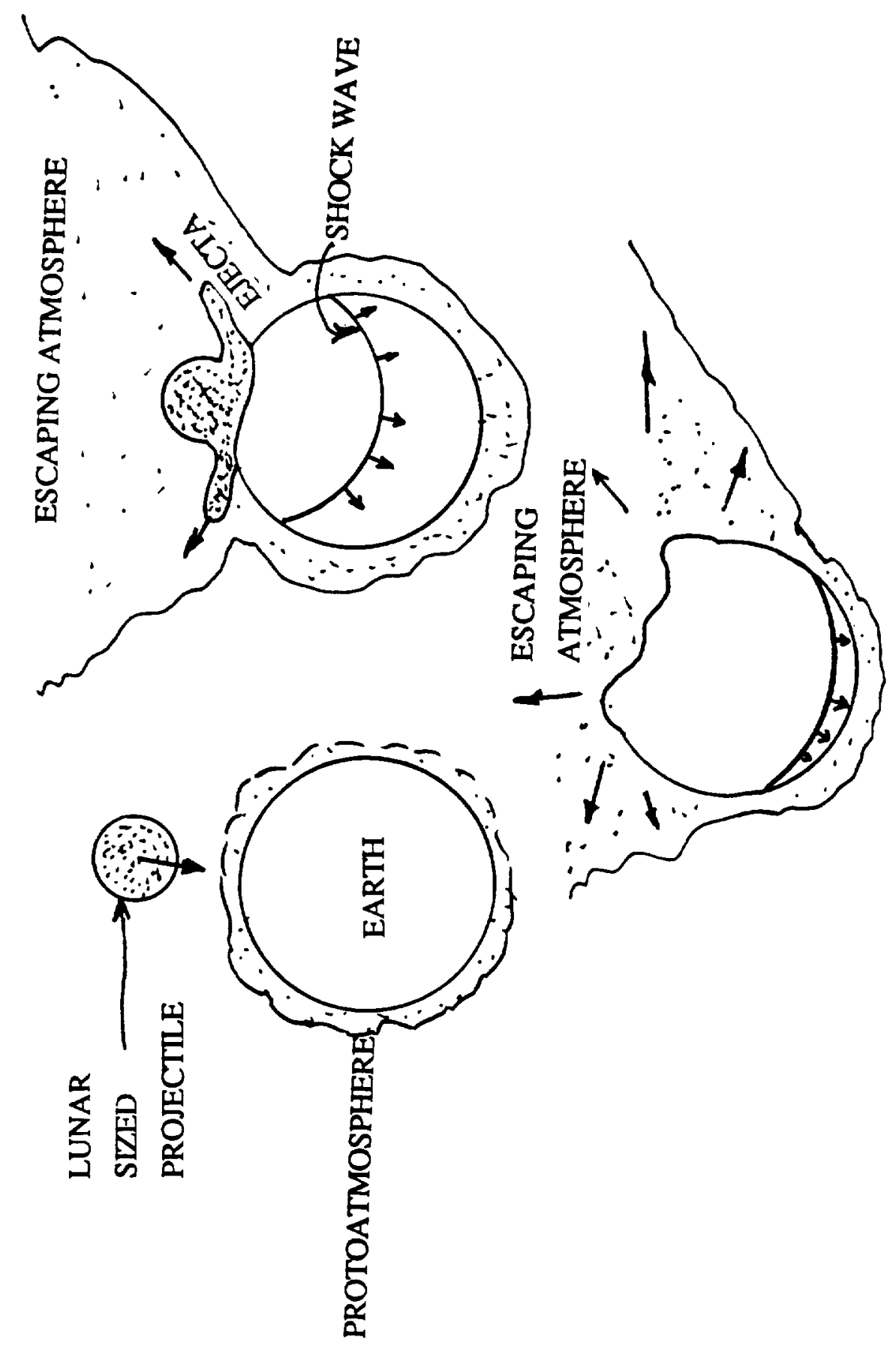




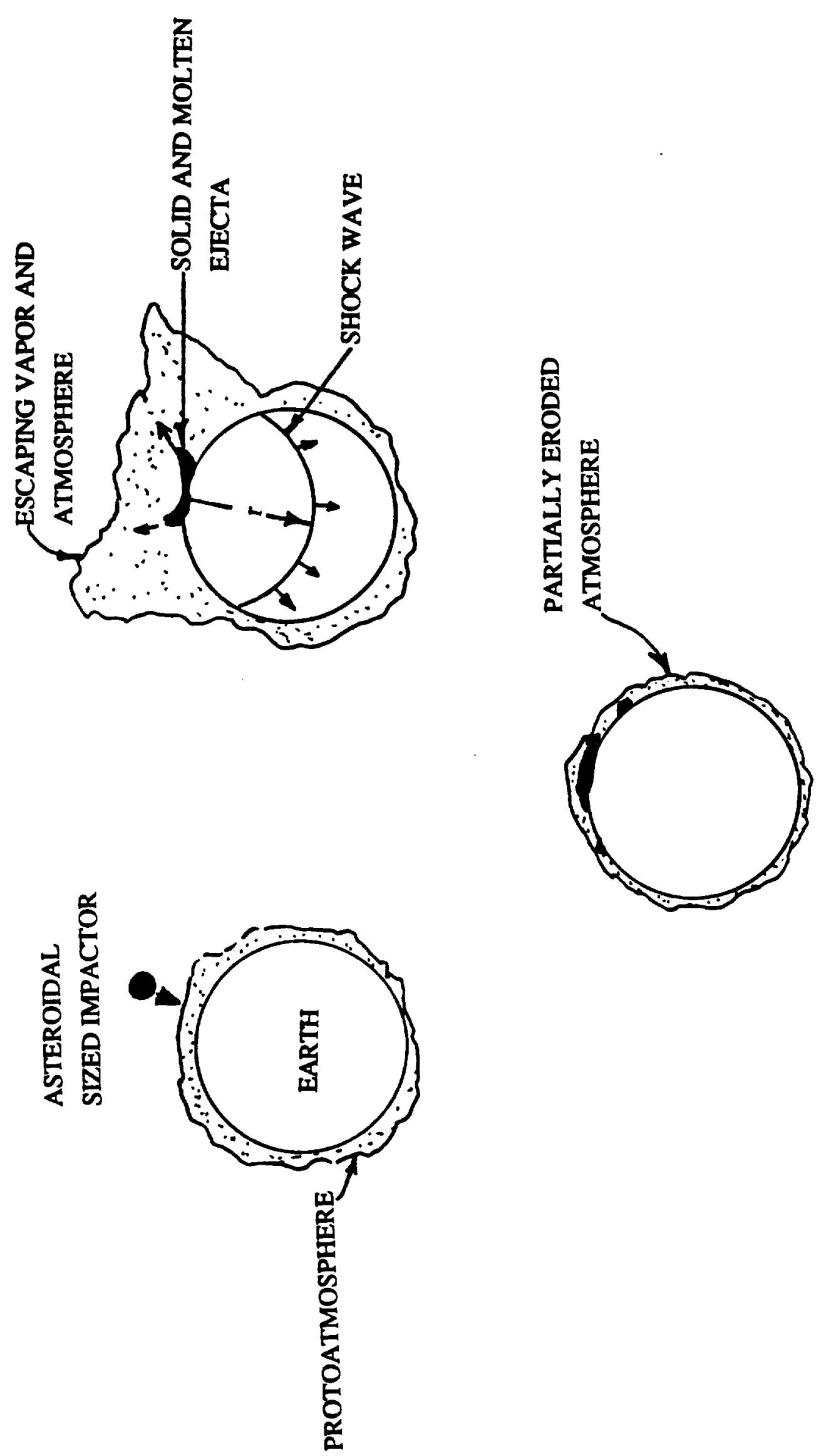

$=$

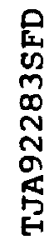

อิ 


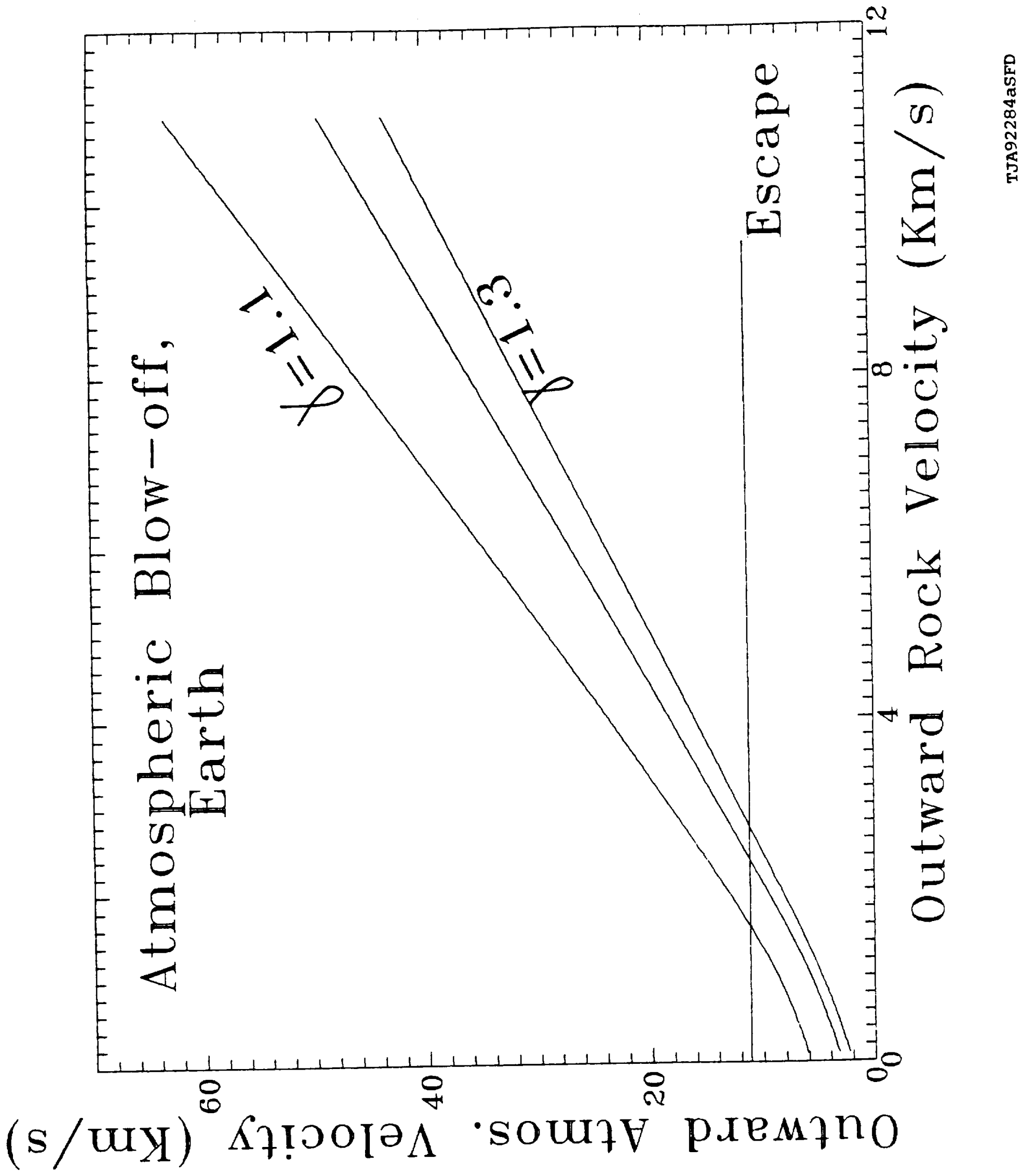




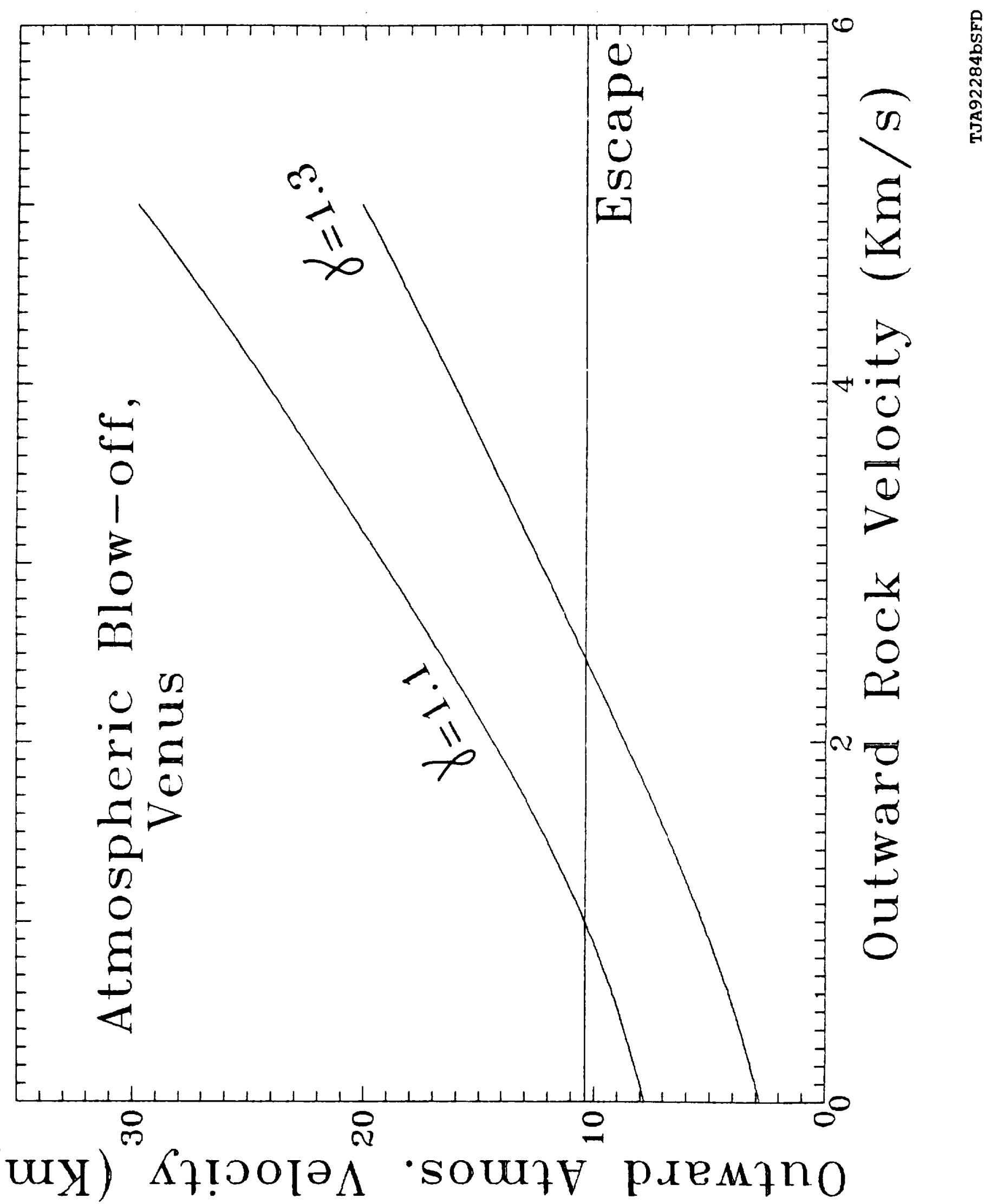




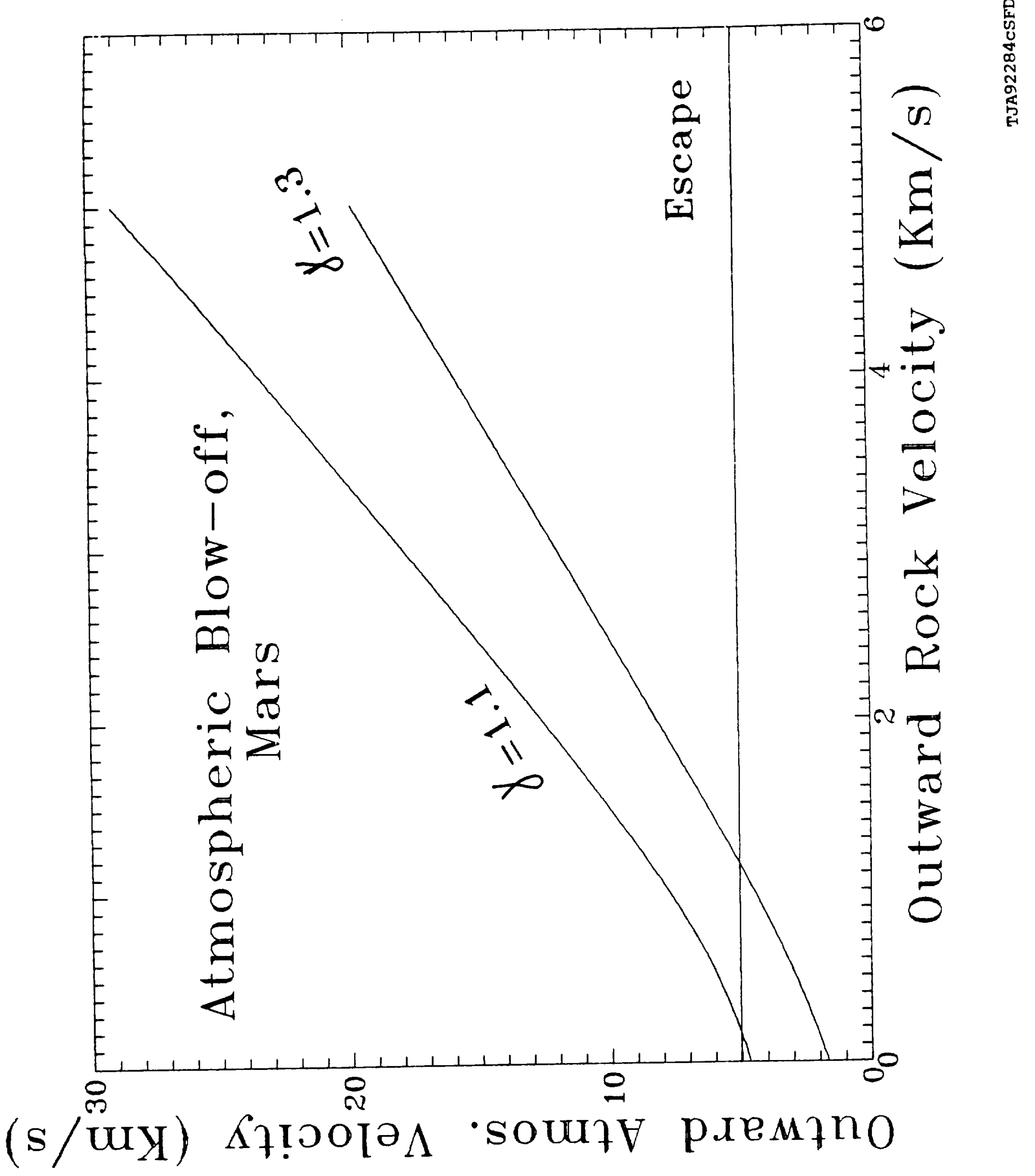




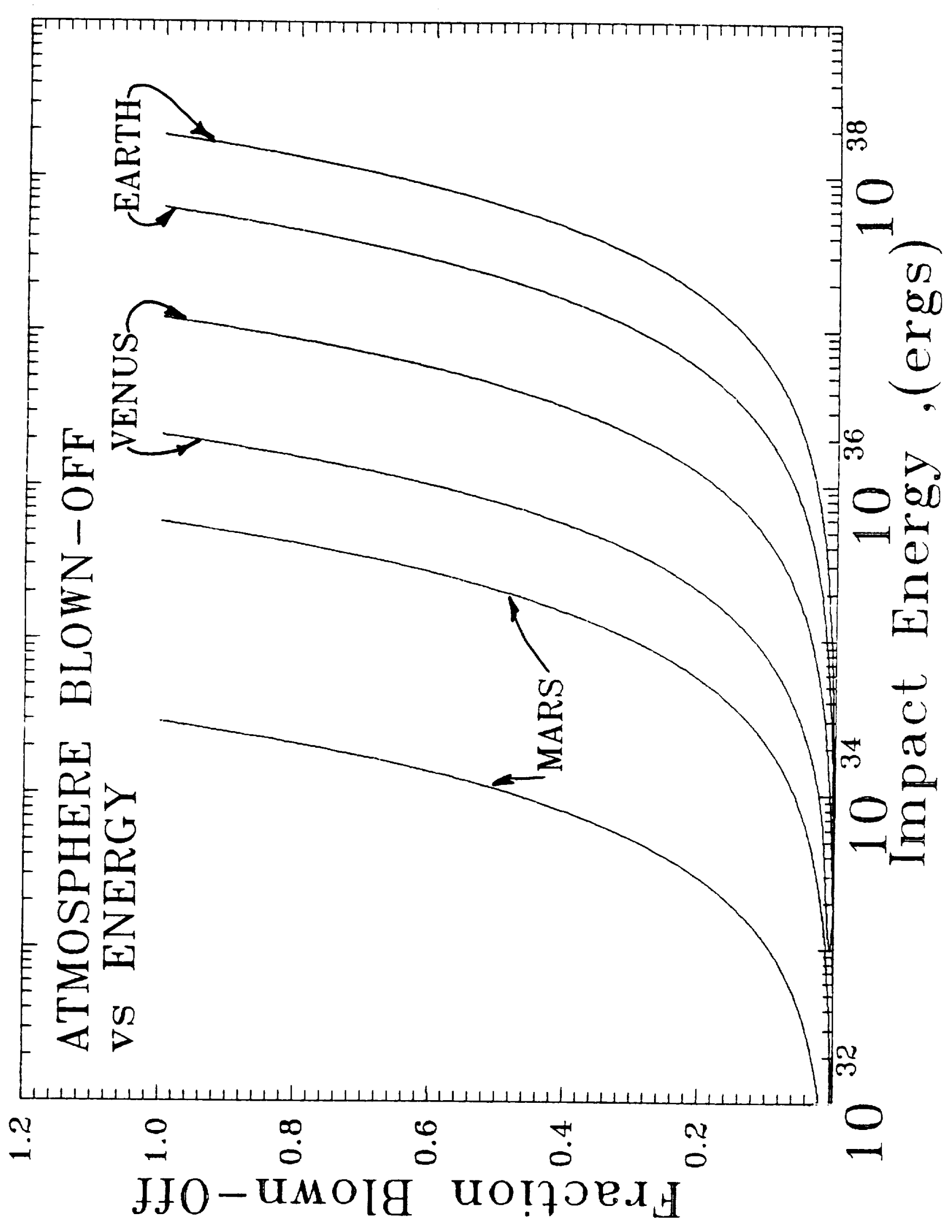

